ARTICLE

\title{
Cross-feeding between intestinal pathobionts promotes their overgrowth during undernutrition
}

\author{
K. E. Huus ${ }^{1,6}$, T. T. Hoang ${ }^{1,6}$, A. Creus-Cuadros ${ }^{1}$, M. Cirstea ${ }^{1}$, S. L. Vogt ${ }^{1}$, K. Knuff-Janzen' ${ }^{1}$, P. J. Sansonetti ${ }^{2,4}$, \\ P. Vonaesch ${ }^{2,5} \&$ B. B. Finlay (D) $1,3 凶$
}

Child undernutrition is a global health issue associated with a high burden of infectious disease. Undernourished children display an overabundance of intestinal pathogens and pathobionts, and these bacteria induce enteric dysfunction in undernourished mice; however, the cause of their overgrowth remains poorly defined. Here, we show that disease-inducing human isolates of Enterobacteriaceae and Bacteroidales spp. are capable of multi-species symbiotic cross-feeding, resulting in synergistic growth of a mixed community in vitro. Growth synergy occurs uniquely under malnourished conditions limited in protein and iron: in this context, Bacteroidales spp. liberate diet- and mucin-derived sugars and Enterobacteriaceae spp. enhance the bioavailability of iron. Analysis of human microbiota datasets reveals that Bacteroidaceae and Enterobacteriaceae are strongly correlated in undernourished children, but not in adequately nourished children, consistent with a diet-dependent growth synergy in the human gut. Together these data suggest that dietary cross-feeding fuels the overgrowth of pathobionts in undernutrition.

\footnotetext{
${ }^{1}$ Michael Smith Laboratories and Department of Microbiology and Immunology, University of British Columbia, Vancouver, BC, Canada. ${ }^{2}$ Unité de Pathogénie Microbienne Moléculaire, Institut Pasteur, Paris, France. ${ }^{3}$ Canadian Institute for Advanced Research, Toronto, Ontario, Canada. ${ }^{4}$ Present address: Center for Microbes, Development and Health, Institut Pasteur de Shanghai, Shanghai, China. ${ }^{5}$ Present address: Human and Animal Health Unit, Swiss Tropical and Public Health Institute \& University of Basel, Basel, Switzerland. ${ }^{6}$ These authors contributed equally: K.E. Huus, T.T. Hoang.

凶email: bfinlay@msl.ubc.ca
} 
$\mathrm{M}$ alnutrition is a global health issue, responsible for nearly half of all deaths in children under 5 years old ${ }^{1,2}$. In addition to diet, an intestinal disorder known as environmental enteric dysfunction (EED) contributes significantly to child undernutrition in regions with inadequate sanitation. EED is characterized by blunting of the small intestinal villi $^{3-6}$ alongside increased intestinal permeability and inflammation ${ }^{7-9}$. EED is thought to be microbially driven and has been linked to dysbiosis and overgrowth of the microbiota, most notably a high burden of Proteobacteria species and asymptomatic carriage of enteropathogens ${ }^{10-13}$. This drives a vicious cycle of nutrient malabsorption and increased susceptibility to infection. However, intervention trials that aimed to provide both an improved diet and improved hygiene in early life failed to reduce the prevalence of bacterial pathogens or to improve stunting or $\mathrm{EED}^{14}$. The etiology of EED and the factors driving microbial dysbiosis in these children remain poorly understood.

In 2015, our lab reported that a combination of dietary malnutrition and specific fecal bacteria could replicate the features of EED in mice ${ }^{15}$. Mice fed an isocaloric low-protein, low-fat diet, and iteratively exposed to commensal human Bacteroidales and $E$. coli isolates displayed impaired growth, small intestinal villous blunting, and abnormal intestinal permeability and inflammation, analogous to human $\mathrm{EED}^{15}$. Another research group independently found that a combination of Bacteroidetes, Enterobacteriaceae, and Enterococcus from children with severe acute malnutrition could induce enteropathy in malnourished gnotobiotic mice ${ }^{16}$. Intriguingly, both models were dependent on the combined presence of both Bacteroidales and Enterobacteriaceae members rather than any taxon individually, suggesting a pathobiotic synergy between these bacteria. Furthermore, both models were dependent on a carbohydrate-rich malnourished diet: mice that received the same bacterial cocktail but were fed an isocaloric control diet rich in protein and fat did not develop $\mathrm{EED}^{15,16}$. The mechanism driving this context-dependent synergy between Bacteroidales and E. coli is currently unknown. We hypothesized that Bacteroidales and E. coli were capable of metabolic cross-feeding and that this would be exacerbated in protein-deficient, carbohydrate-rich environments, leading to synergistic growth of these bacteria and intestinal dysbiosis in EED mice.

Metabolic interactions between Bacteroidales and Enterobacteriaceae strains have been previously shown to contribute to microbial dysbiosis and to the outgrowth of pathogens. Bacteroides spp. digest complex polysaccharides in the host diet and host mucosa, liberating simple sugars that act both as growth substrates and as signaling cues for pathogenic E. coli and Salmonella ${ }^{17-20}$. Conversely, Bacteroides spp. have been shown to benefit from the presence of Enterobacteriaceae during intestinal and extraintestinal infections, thanks to the production of hemoglobin-degrading proteases and of iron-scavenging siderophores by Enterobacteriaceae ${ }^{21,22}$. However, both of these interactions were studied independently, and reciprocal crossfeeding in mixed communities has not been shown. It is further unknown how these interactions are influenced by diet.

Here, we show that EED-inducing strains of Bacteroidales spp. and Escherichia spp. are capable of mutual, bidirectional crossfeeding, resulting in synergistic growth of a mixed community in vitro. Growth synergy is highly dependent on the nutritional environment and requires the presence of both host mucin and dietary carbohydrates under protein- and iron-limiting conditions. Moreover, the abundances of Bacteroidaceae and Enterobacteriaceae are strongly correlated in the fecal microbiota of undernourished, but not normally nourished, children. Together these data indicate a role for host nutrition in shaping pathobiont co-dependencies within the microbiota.

\section{Results}

Synergistic growth of Bacteroidales and $E$. coli in co-culture. We designed a complex medium, designated "MAL-M", to reflect the macronutrients available in the intestine during chronic protein undernutrition. Dietary plant polysaccharides (starch, inulin, and cellulose) and protein (casein) were included based on their abundance in a malnourished mouse diet previously reported by our group ${ }^{15}$, and mucin was added as a source of host glycoprotein (Fig. 1a and Supplementary Table 1), leading to a medium rich in carbohydrates but deficient in protein and fat. This medium was used to co-culture seven human intestinal isolates used previously to induce inflammation and barrier disruption in our mouse model of malnutrition and EED: five strains of Bacteroidales (Bacteroides fragilis, B. vulgatus, B. ovatus, B. dorei, Parabacteroides distasonis) and two Enterobacteriaceae (Escherichia coli 4_1_47 and Escherichia coli 3_2_53 (Fig. 1a and Supplementary Table 2). Unless otherwise indicated, in this manuscript Bacteroidales will refer to all five strains of Bacteroidales grown in combination, while $E$. coli will refer to both strains of $E$. coli grown together ("B" and "E", respectively, in the figures; Fig. 1a).

Both Bacteroidales and $E$. coli were capable of growing in MAL-M, attaining $10^{7}$ and $10^{8} \mathrm{CFU} / \mathrm{mL}$, respectively, after $24 \mathrm{~h}$ of anaerobic growth (Fig. $1 \mathrm{~b}$ and Supplementary Fig. 1). When all seven species were grown in combination, the co-culture grew to $10^{9}$ total $\mathrm{CFU} / \mathrm{mL}$, a tenfold increase that was substantially more than expected based on simple additive growth (Fig. 1b).

The synergistic increase in bacterial CFU was driven by improved growth of both Bacteroidales and E. coli simultaneously. The growth of $E$. coli roughly doubled in the presence of Bacteroidales (Fig. 1c), while the growth of Bacteroidales increased by tenfold or more in the presence of E. coli (Fig. 1d). These bacteria thus mutually support one another's growth through cooperative interactions.

Synergistic growth is dependent on the nutritional environment. To better understand the importance of the nutritional environment in promoting Bacteroidales-E. coli growth synergy, we compared growth in the original MAL-M medium with medium in which the weight-based ratio of protein to carbohydrate was reversed (“CON-M"). Bacteria were also grown in CON and MAL media in the absence of host mucin (Supplementary Fig. 2A and Supplementary Table 1). In these complex media, the presence of mucin promoted the total growth of the co-culture (Supplementary Fig. 2B). However, only the carbohydrate-rich mucin medium (MAL-M) led to enhanced growth of both $\mathrm{Bac}$ teroidales and $E$. coli simultaneously compared to their respective monocultures (Supplementary Fig. 2C, D).

Substitution of casein with whey as the protein source in MAL$\mathrm{M}$ media also resulted in similar reciprocal growth advantages for both Bacteroidales and E. coli (Supplementary Fig. 2E, F). To more clearly distinguish the importance of carbohydrate versus protein in promoting synergistic growth, we cultured bacteria in media containing only dietary carbohydrates (inulin, starch, and cellulose; "Carb") or only dietary protein (casein; "Prot") as the carbon source, with or without host mucin (Fig. 2a and Supplementary Table 1; "CarbM" and "ProtM" designate mucincontaining media). Importantly, when grown separately, Bacteroidales and E. coli each showed similar growth in the CarbM compared to the ProtM media, indicating that the inherent availability of nutrients is comparable between these two media (Fig. 2b-d). However, total bacterial counts in co-culture were significantly higher in the carbohydrate- and mucus-rich CarbM medium than in any other condition, with both E. coli and Bacteroidales showing a distinct advantage in CarbM co-culture compared to ProtM co-culture (Fig. 2b-d). 
(a)

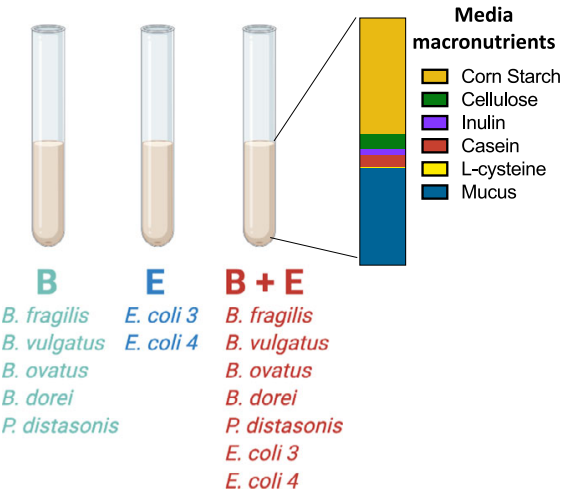

(c)

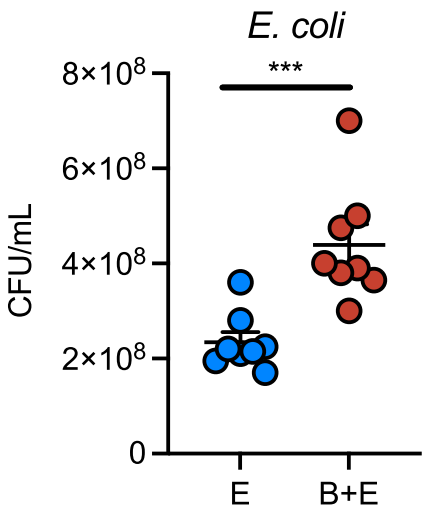

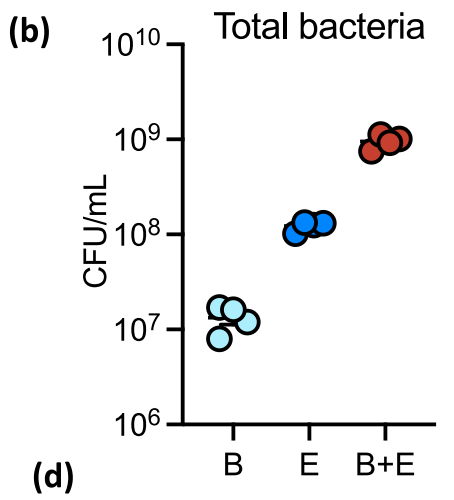

(d)

Bacteroidales

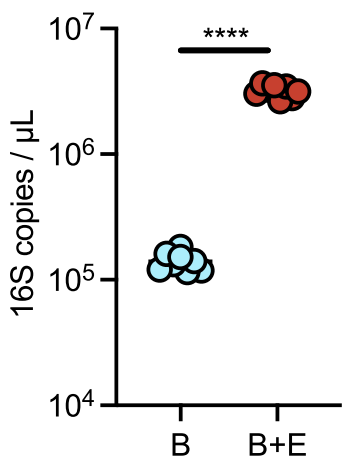

Fig. 1 Synergistic growth of Bacteroidales and Enterobacteriaceae in co-culture. a Schematic of the experimental set-up. After a $24 \mathrm{~h}$ culture, bacterial endpoint growth was determined for $(\mathbf{b})$ the total community $(n=4)$, c E. coli $(n=8)$, and $\mathbf{d}$ Bacteroidales $(n=8)$. Mean $+/-$ SEM are displayed. Significance was determined by two-sided $t$ test. ${ }^{\star \star \star} P<0.001 ;{ }^{\star \star \star \star} P<0.0001$. c, d B Bacteroidetes mix, E E. coli mix, CFU colony-forming units. Source data are provided as a Source Data file. Exact $P$ values and test statistics are reported in Supplementary Table 8.

Iron is a key micronutrient that is frequently limited in undernourished diets, and Bacteroidales are known to be particularly dependent on organic iron sources ${ }^{23}$. To investigate the impact of iron bioavailability, we cultured bacteria in the original MAL-M medium (containing $1.9 \mu \mathrm{M}$ hematin) or in a medium where organic iron was provided at tenfold excess (19 $\mu \mathrm{M}$ hematin). Bacteroidales, but not E. coli, grew substantially better in the hematin-enriched medium (Fig. 2e, f). Strikingly, however, excess hematin significantly dampened the synergistic advantage of both Bacteroidales and E. coli when grown in coculture (Fig. 2e, f). Although the hematin was dissolved in a histidine solution to improve its stability and bioavailability, a control condition supplemented with histidine only did not impact Bacteroidales growth (Supplementary Fig. 2G). These data suggest that organic iron is a limiting nutrient for Bacteroidales growth in MAL-M medium, and that iron bioavailability affects synergistic growth in co-culture.

Together, these data support the importance of the nutritional environment in Bacteroidales-E. coli synergism. Bacteroidales and E. coli experience a mutual growth advantage under protein- and iron-limited conditions in the presence of mucus and dietary carbohydrates.

Competitive and cooperative interactions contribute to strain abundance in the mixed community. To determine whether the expansion of Bacteroidales was driven by the abundance of one or more of the individual species present in our cocktail, we performed 16S rRNA sequencing of Bacteroidales cultures in MAL-M media in the presence or absence of E. coli. Relative abundances were converted to total abundances by means of Bacteroidetes-specific 16S rRNA qPCR. All five Bacteroidales strains were able to grow in co-culture in the absence of $E$. coli (Supplementary Fig. 3A-E). However, B. fragilis was by far the dominant taxon by relative abundance; moreover, in the presence of E. coli, B. fragilis bloomed at the expense of the other Bacteroides (Fig. 3a and Supplementary Fig. 3A-E). Indeed, after $24 \mathrm{~h}$ of growth in co-culture, B. fragilis constituted over $50 \%$ of the Bacteroidales by relative abundance and $B$. vulgatus was undetectable. Community richness and evenness, as represented by the Shannon index, were thus significantly decreased in co-culture by $24 \mathrm{~h}$, despite the greater initial diversity of this mixture (Fig. 3b). Notably, although $B$. ovatus relative abundance was decreased in the presence of $E$. coli by $24 \mathrm{~h}$ (Fig. 3a), B. ovatus showed only a mild defect in total abundance at $24 \mathrm{~h}$ (Supplementary Fig. 3C), and in fact showed improved total growth in the presence of $E$. coli at the earlier $16 \mathrm{~h}$ timepoint, suggesting it might simply experience an earlier benefit than the other strains.

To further delineate strain interactions, we tested growth pairs of $E$. coli with each individual Bacterodales spp. In contrast to the results from a mixed culture, all individual Bacteroidales benefited substantially from pairwise co-culture with E. coli (Fig. 3c). This suggests that all strains tested here are capable of cross-feeding with E. coli, but that competition with other Bacteroidales restricts this benefit in more complex communities. Indeed, when growth pairwise with $E$. coli, both $B$. fragilis and B. ovatus were capable of reaching similar total biomass to the entire Bacteroidales coculture (Fig. 3c).

Reciprocally, all Bacteroides strains appeared to confer a modest growth advantage to $E$. coli in pairwise co-culture, but to varying degrees (Fig. 3d). There was notable batch variation in the magnitude of bacterial growth, possibly due to media batch effects or minor timing differences; however, trends were consistent 
(a)

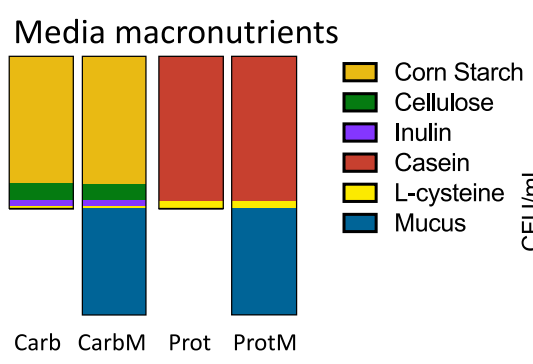

Carb CarbM Prot ProtM

(c)

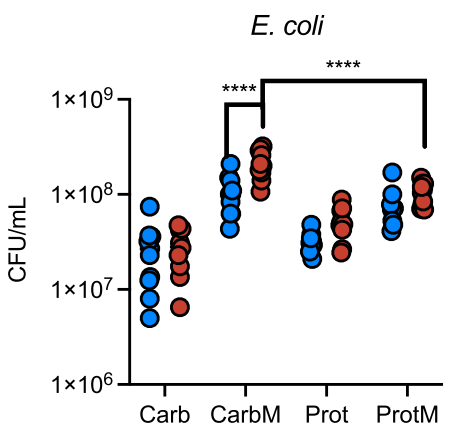

(e)

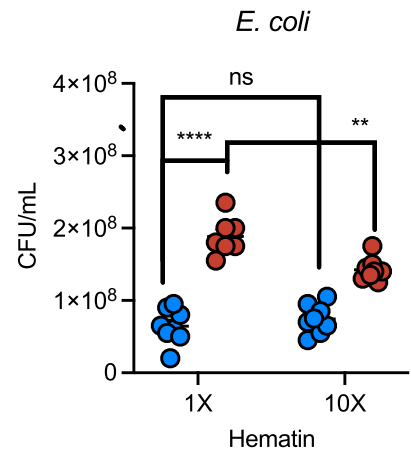

(b)

Total bacteria

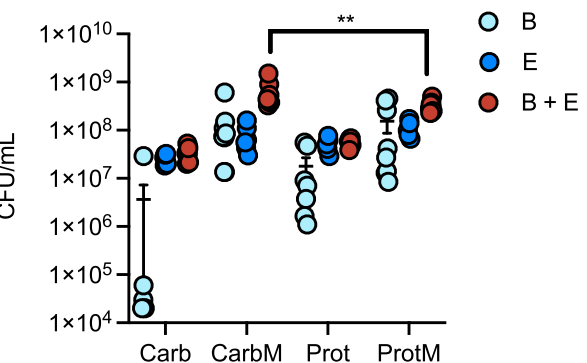

(d)

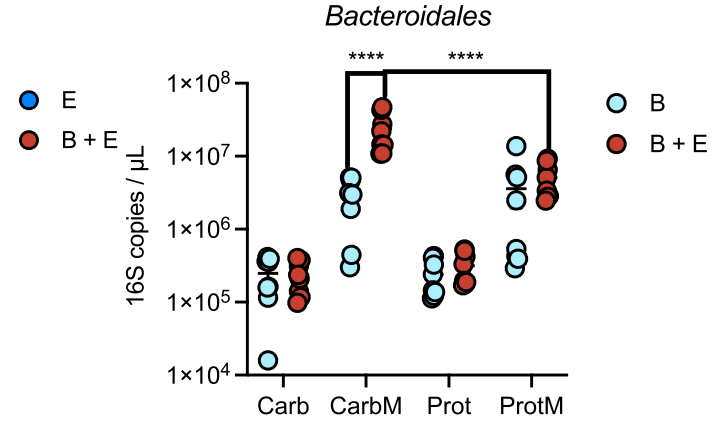

(f)

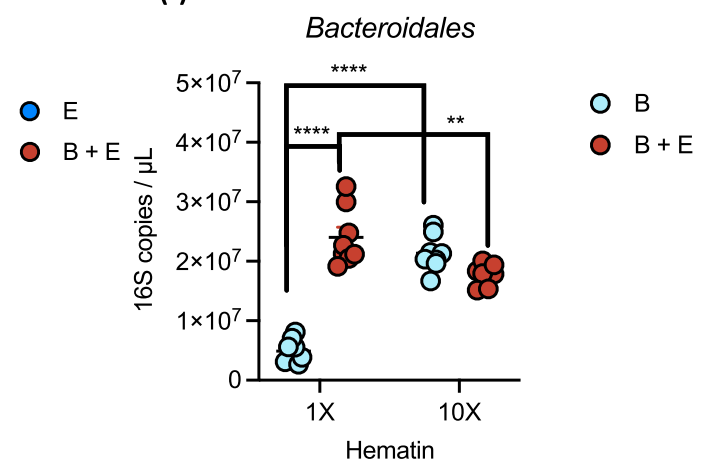

Fig. 2 Synergistic growth of Bacteroidales and Enterobacteriaceae is dependent on the nutritional environment. a Media macronutrient compositions. After a $24 \mathrm{~h}$ culture, bacterial endpoint growth was determined for (b) the total community, $\mathbf{c} E$. coli and $\mathbf{d}$ Bacteroidetes $(n=8)$. e, $\mathbf{f}$ Growth of $(\mathbf{e})$ E. coli and (f) Bacteroidales in MAL-M media with $1 \times(1.9 \mu \mathrm{M})$ or $10 \times(19 \mu \mathrm{M})$ hematin $(n=8)$. Mean $+/-\mathrm{SEM}$ are displayed. Significance was determined by twoway ANOVA with Tukey's post hoc test $(\mathbf{b}-\mathbf{f})$. ${ }^{\star \star} P$ value $<0.01,{ }^{\star \star \star \star} P$ value $<0.0001$. B Bacteroidales mix, E E. coli mix, CFU colony-forming units, Carb carbohydrate media, CarbM carbohydrate plus mucus media, Prot protein media, ProtM protein plus mucus media. Source data are provided as a Source Data file. Exact $P$ values and test statistics are reported in Supplementary Table 8.

between replicates (Supplementary Fig. 3F-H). B. ovatus provided the most consistent and significant individual growth benefit to E. coli, largely recapitulating the effect of the complete community (Fig. 3d and Supplementary Fig. 3F-H). Nevertheless, removing $B$. ovatus from the complete mix still resulted in a substantial co-culture benefit for E. coli (Supplementary Fig. 3H), suggesting that the contributions of the other taxa are sufficient in combination to provide a growth advantage to $E$. coli.

Together these data indicate that many different members of the Bacteroidales order are capable of cooperation with E. coli during nutrient-limited conditions. In the presence of other Bacteroidales, both cooperation and competition contribute to the emergent growth of the community.

Bacteroidales and $E$. coli exchange soluble metabolites. To determine whether growth synergism in co-culture was mediated by soluble factors, we assessed bacterial growth in sterile supernatants from blank media or from 24-h cultures of Bacteroidales or E. coli in MAL-M. As expected, E. coli grew significantly better in supernatants from Bacteroidales culture compared to blank media or its own spent growth media, while Bacteroidales grew significantly better in $E$. coli culture supernatants compared to blank media or its own spent growth media (Fig. $4 \mathrm{a}, \mathrm{b}$ and Supplementary Fig. 4A, B). Thus, both E. coli and Bacteroidales produce soluble factors in carbohydrate-rich environments that benefit the other community member.

To identify metabolic pathways involved in synergistic growth, we began by testing the role of mucin-derived sugars, since mucus stimulated co-culture growth (Fig. 2b and Supplementary Fig. 2B) and has been previously shown to fuel microbial crossfeeding ${ }^{17,18}$. Indeed, co-culture of $E$. coli with $B$. thetaiotaomicron, a species previously shown to cooperatively liberate sialic acid from mucus ${ }^{17}$, gave a dramatic growth benefit to these $E$. coli strains (Supplementary Fig. 4C). We, therefore, generated knockout strains of $E$. coli lacking key metabolic enzymes for consumption of sialic acid $(\Delta n a n A)$ and fucose $(\Delta f u c I)$, two sugars that decorate mucin (Supplementary Table 2). These mutant strains were completely unable to grow on sialic acid or fucose, respectively (Supplementary Fig. 4D, E) but showed a growth advantage compared to wild-type $E$. coli on glucose 


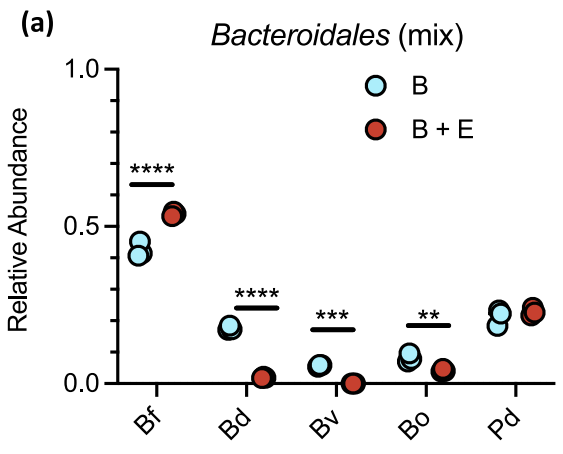

(b)
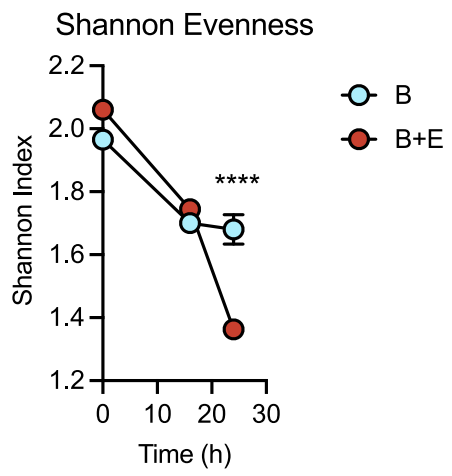

(c)

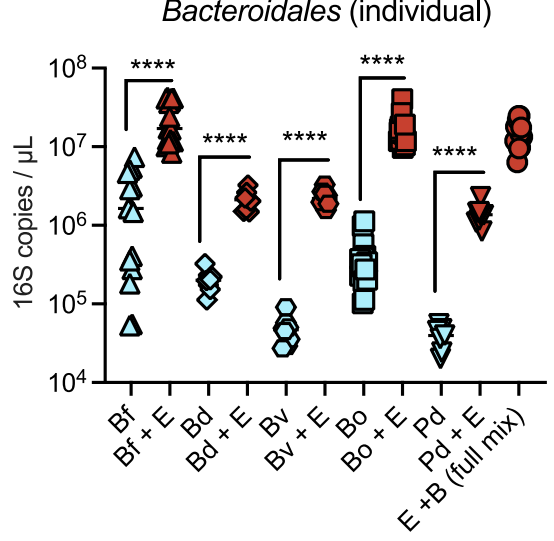

(d)

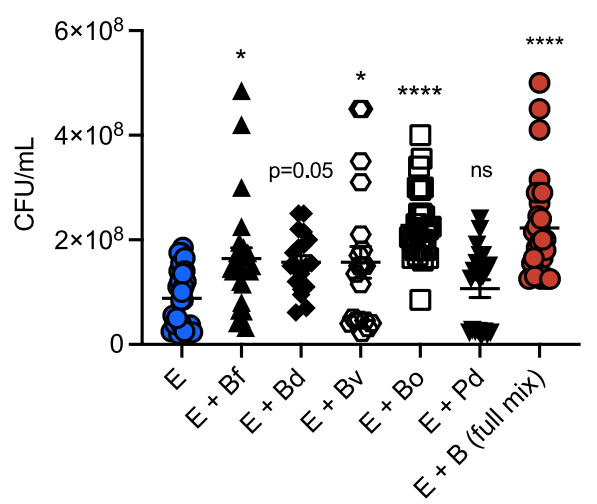

Fig. 3 Competitive and cooperative interactions contribute to strain abundance in the mixed community. a Relative abundance of each Bacteroidales species at $24 \mathrm{~h}$ endpoint, in a mixed community with or without $E$. coli $(n=3)$. b Shannon evenness of the communities over time ( $n=3)$. c $\mathrm{Growth}$ of each individual Bacteroidales at $24 \mathrm{~h}$ endpoint, alone or in co-culture with $\mathrm{E}$. coli ( $n=8$ for Bd, Bv, $\mathrm{Pd} ; n=15$ for Bf, Bo, $\mathrm{E}+\mathrm{B})$. d Growth of $E$. coli at $24 \mathrm{~h}$ endpoint with or without each individual Bacteroidales species or the full mix of all five Bacteroidales ( $n=20$ for Bd, Bv, Bd; $n=27$ for Bf, Bo, E $+B$ ). Mean $+/-\mathrm{SEM}$. Significance was determined by two-way ANOVA with Sidak's post hoc test (a, b) or Dunnett's post hoc test (d). In (c) each strain comparison was independent of the others; significance was determined by repeated two-sided $t$ test with FDR correction. ${ }^{\star} P$ value $<0.05,{ }^{\star \star} P$ value $<0.01$, ${ }^{\star \star \star} P$ value $<0.001,{ }^{\star \star \star \star} P$ value $<0.0001$. B full Bacteroidales mix, E E. coli mix, Bf B. fragilis, Bo B. ovatus, Bv B. vulgatus, Pd P. distasonis, Bd B. dorei. Source data are provided as a Source Data file. Exact $P$ values and test statistics are reported in Supplementary Table 8.

(Supplementary Fig. 4F), possibly because there is a metabolic cost to producing these enzymes in the absence of a substrate.

E. coli $\triangle$ nanA strains were at a significant competitive disadvantage compared to wild-type $E$. coli in MAL-M media during Bacteroidales co-culture, but were not at a disadvantage when grown alone (Fig. 4c). Further supporting the importance of carbohydrate-rich nutritional context that we identified earlier, when $\triangle$ nanA E. coli was co-cultured in the Carb and Prot media described above, we only observed a significant competitive defect of $\triangle n a n A$ E. coli in CarbM media (Supplementary Fig. 4G). There was no significant competitive defect for $\Delta n a n A$ in ProtM media, even though this condition contained identical quantities of mucin as a source of sialic acid, which is consistent with the lack of co-culture growth advantage in this media (Fig. 2b-d). We did not observe any competitive defect for the $\Delta f u c I$ mutant in coculture (Supplementary Fig. $4 \mathrm{H}$ ). Together these data suggest that sialic acid metabolism contributes to $E$. coli growth in the presence of Bacteroidales, and that this interaction is specifically enhanced in a polysaccharide- and mucin-rich environment.

$B$. fragilis has been shown to benefit from E. coli during extraintestinal co-infection due to E. coli liberation of heme from hemoglobin $^{21}$. E. coli also produces siderophores which can be co-opted by Bacteroides for iron acquisition ${ }^{22}$ and E. coli supports porphyrin-auxotrophic Bacteroides in co-culture ${ }^{24}$. We confirmed that the E. coli strains used in this study also produce siderophores under iron-limiting conditions (Supplementary
Fig. 5A). We further investigated the importance of iron using $B$. fragilis as a proof-of-principle strain. Similar to total Bacteroidales growth, the growth of $B$. fragilis was significantly enhanced by hematin supplementation (Supplementary Fig. 5B). Growth was not dependent on the concentration of vitamin B12, another putatively limiting micronutrient (Supplementary Fig. 5C). To further test whether iron was responsible for the $B$. fragilis growth advantage in co-culture, we used a $B$. fragilis $\triangle$ feo $A B$ knockout strain deficient in both ferrous iron and heme uptake ${ }^{25}$ and compared it to parent strain B. fragilis 638R (Supplementary Table 2). Similar to the $B$. fragilis strain $3 / 1 / 12$ used elsewhere in this paper (and indeed to all Bacteroides spp. tested here), $B$. fragilis 638R experienced a significant growth advantage in MAL$\mathrm{M}$ media in the presence of E. coli (Supplementary Fig. 5D). Further, $\triangle f e o A B B$. fragilis was at a competitive disadvantage compared to its parent strain, but only when co-cultured in the presence of E. coli (Fig. 4d). These data suggest that B. fragilis benefits from $E$. coli-derived iron.

Both $\triangle$ nanA E. coli and $\triangle f e o A B B$. fragilis still benefited from co-culture when grown without a wild-type competitor, suggesting that other unidentified factors also contribute to growth synergy (Supplementary Fig. 5D, E). To identify other potential nutrient sources in co-culture, we quantified a targeted panel of saccharides and short-chain fatty acids in bacterial supernatants using mass spectrometry. In addition to the complex mixes of Bacteroidales, E. coli, and co-cultured bacteria, we included 
$\begin{array}{llll}\text { (a) } & \text { E. coli } & \text { (b) Bacteroidales (c) E. coli } \triangle \text { nanA:WT (d) B. fragilis } \triangle \text { feoAB:WT }\end{array}$
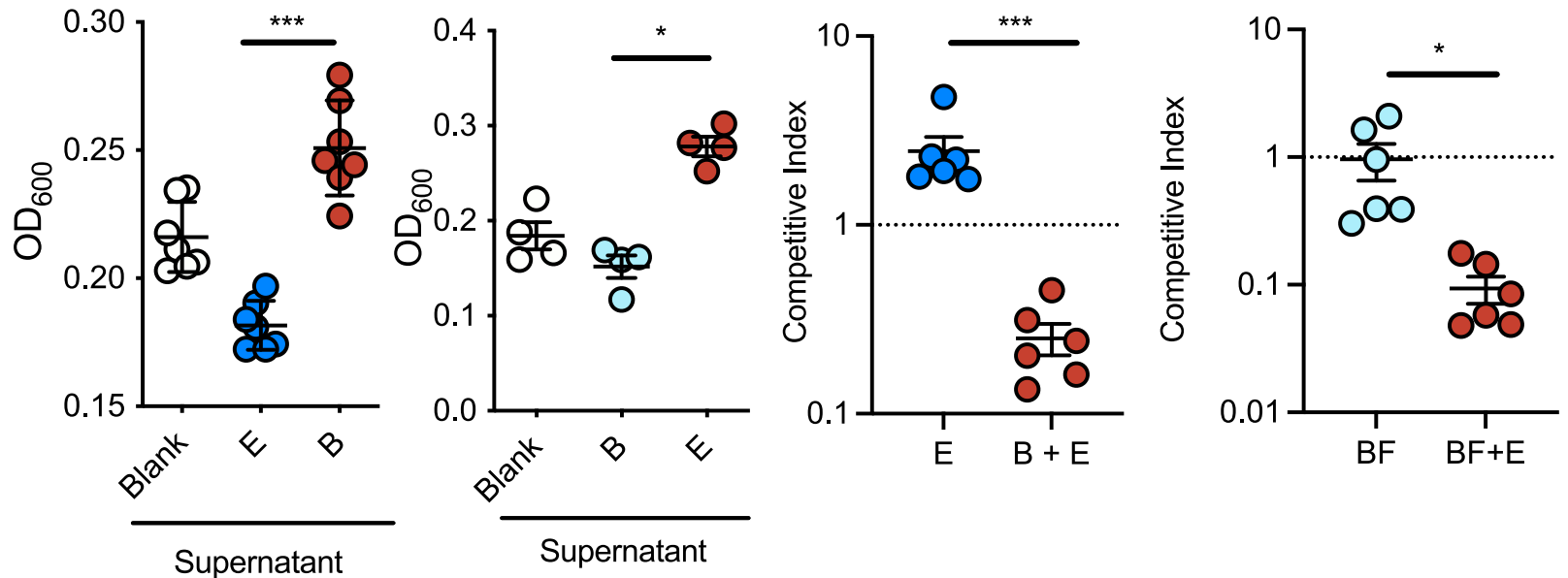

(e)

GlcNAc

(f)

Supernatant
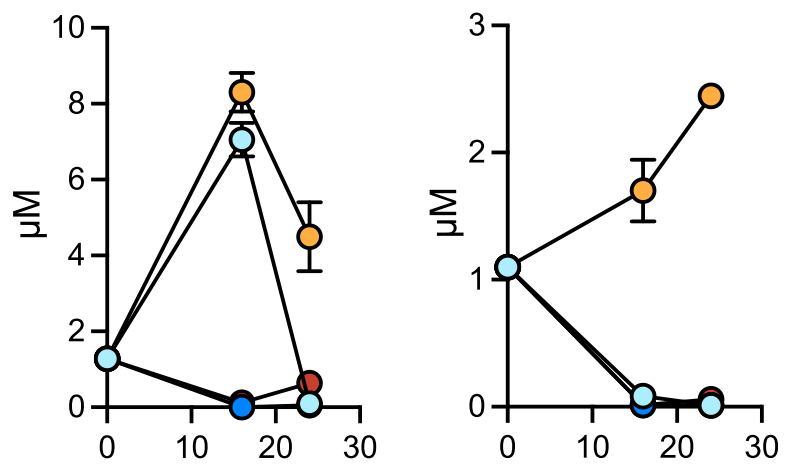

(g)

Maltose

(h)

Fructose
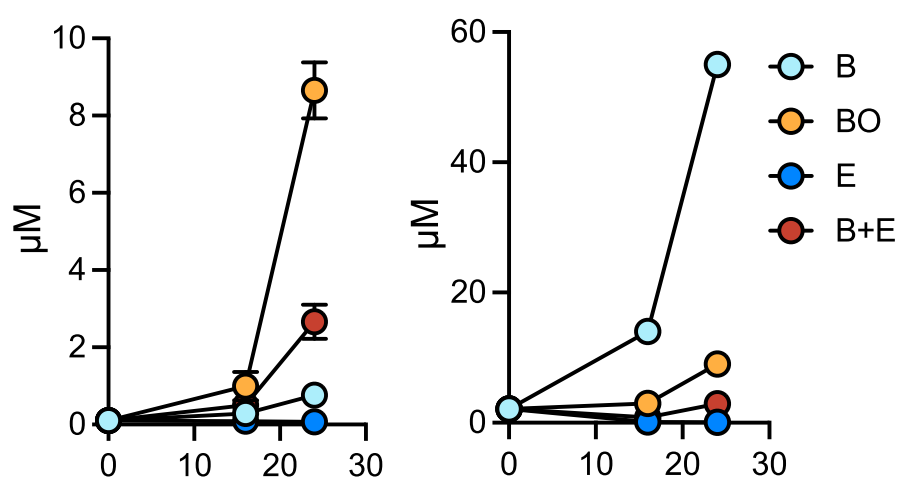

(i)

(j)

(k)
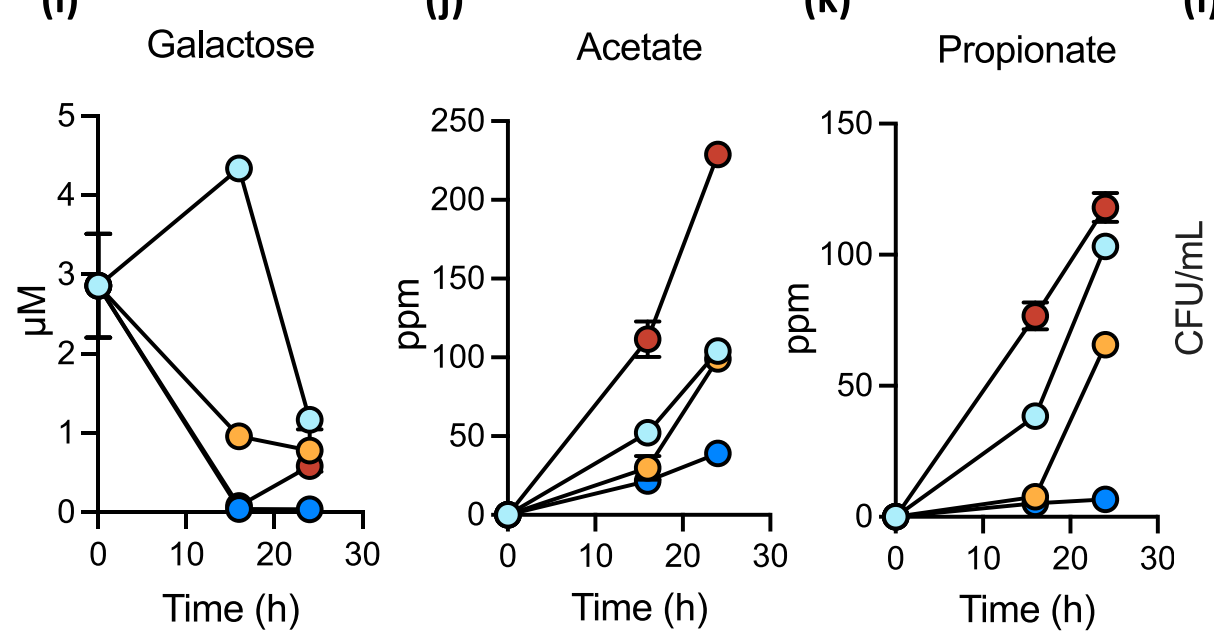

(I)

E. coli

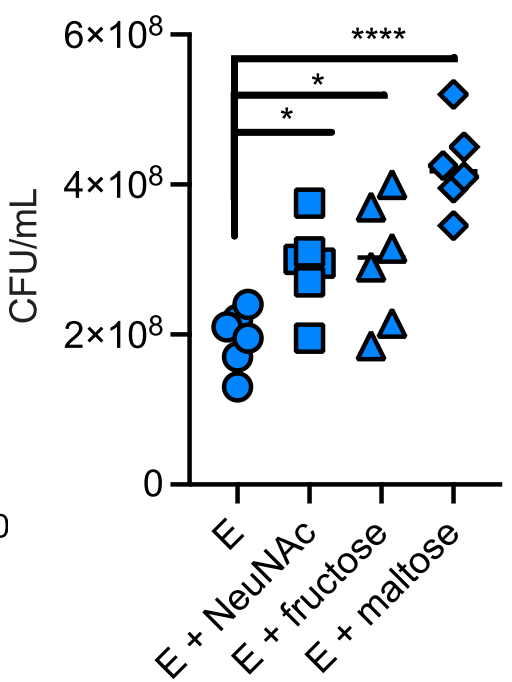

Fig. 4 E. coli and Bacteroidales exchange soluble metabolites, including sialic acid and heme. a, b Growth of $E$. coli $(n=7)(\mathbf{a})$ and Bacteroidales $(n=4)$ (b) at 24 endpoint, as determined by optical density at $600 \mathrm{~nm}$ (O.D.600) in the sterile-filtered supernatants of blank media ("Blank"), a $24 \mathrm{~h}$ E. coli culture ("E"), or a $24 \mathrm{~h}$ Bacteroidales culture ("B"). c Competitive index of $\triangle$ nanA E. coli, deficient in sialic acid metabolism, compared to wild-type in the presence or absence of Bacteroidales $(n=6)$. d Competitive index of $\triangle$ feoAB B. fragilis, deficient in iron uptake, compared to wild-type strain $638 \mathrm{R}$ in the presence or absence of $E$. coli $(n=6)$. e-k Metabolite abundance in sterile-filtered bacterial culture supernatants over time $(n=3)$. Mean $+/-$ SEM are displayed: e N-acetylglucosamine (GIcNAc); $\mathbf{f} \mathrm{N}$-acetylneuraminic acid (NeuNAc, sialic acid); $\mathbf{g}$ maltose; $\mathbf{h}$ fructose; $\mathbf{i}$ galactose; $\mathbf{j}$ acetate; $\mathbf{k}$ propionate. I Growth of E. coli in MAL-M media supplemented with $1 \mathrm{mM}$ of free sugars $(n=6)$. Significance was determined by ANOVA with post hoc Dunnett's test (a, $\mathbf{b}$, I), or by two-sided $t$ test $(\mathbf{c}, \mathbf{d}) .{ }^{\star} P$ value $<0.05,{ }^{\star \star} P$ value $<0.01,{ }^{\star \star \star} P$ value $<0.001$. B Bacteroidales mix, BF B. fragilis, BO B. ovatus, E E. coli mix. Source data are provided as a Source Data file. Exact $P$ values and test statistics are reported in Supplementary Table 8. 
B. ovatus as a proof-of-principle strain due to its ability to provide a substantial individual benefit to E. coli (Fig. 3d). These results indicate that a large number of mucin- and carbohydrate-derived sugars are generated by the full Bacteroidales mix, including $\mathrm{N}$-acetylglucosamine, fructose, galactose, xylose, and fucose (Fig. 4e-i and Supplementary Fig. 6A-L). $B$. ovatus was independently capable of generating many of these same sugars and was also a net producer of sialic acid and maltose (Fig. 4e-g). Importantly, all of these sugars were depleted in E. coli monoculture relative to baseline, suggesting that E. coli consumes them; further, they were largely depleted in Bacteroidales-E. coli co-culture (Fig. 4e-i and Supplementary Fig. 6A-L). In contrast, the short-chain fatty acids acetate and propionate, two end products of microbial fermentation, accumulated in Bacteroidales-E. coli co-culture (Fig. 4j, k). The growth of E. coli was enhanced in MAL-M media supplemented with free sialic acid, fructose, or maltose, supporting the ability of any or all of these sugars to provide a growth advantage to E. coli during cross-feeding (Fig. 4l).

Together these data identify a large number of possibilities for carbohydrate-based cross-feeding between Bacteroidales and E. coli, and suggest efficient utilization of shared resources in the mixed community.

Bacteroidaceae and Enterobacteriaceae are co-abundant in the fecal microbiota of undernourished children. We hypothesized that if the interbacterial synergism between Bacteroidales and Enterobacteriaceae occurs in the human intestine, members of these taxa should be co-abundant in the microbiota of undernourished children, whose diets are typically rich in carbohydrates but poor in sources of animal protein. To test this prediction, we analyzed 16S rRNA sequencing data from fecal samples of children in low- and middle-income countries. Data were retrieved from four studies spanning five countries (Central African Republic, India, Madagascar, Malawi, and Peru) and included publicly available data as well as new sequences generated through the Afribiota study ${ }^{11,12,26-28}$. Children were included for analysis if they were 2-5 years of age and had linear growth measurements available as an outcome. Linear growth stunting, a reflection of chronic undernutrition, was defined by height-for-age z-score as detailed in "Methods".

Consistent with our prediction, the relative abundance of the Bacteroidaceae and Enterobacteriaceae families were significantly positively correlated in the microbiota of stunted children, but not of nonstunted children (Table 1 and Fig. 5a). Moreover, the association between these taxa became progressively stronger with degree of stunting severity (Fig. 5b), supporting the importance of nutritional status in modulating this association. The correlation between Bacteroidaceae and Enterobacteriaceae in stunted children was consistent in four out of the five countries individually, as well as in the pooled analysis (Table 1); the only exception was the dataset from Dinh et al. ${ }^{28}$, where the sample size after exclusion was very small $(n=8)$. The distribution of stunting was not different between the studies $(P>0.1$ by ANOVA), and the pooled analysis in stunted children remained significant in a linear model correcting for study effect $(P=0.037)$. Age was also not different between stunted and nonstunted children $(P>0.1$ by $t$ test) and the taxa correlation remained significant in a linear model correcting for both study effect and age $(P=0.039)$.

In Madagascar and the Central African Republic, upper intestinal (duodenal and gastric) samples from stunted children were additionally sequenced; the taxonomic correlations in this intestinal site were much less striking (duodenal: $n=140$, $P=0.0958$, rho $=0.14$; gastric: $n=61, P=0.3493$, rho $=0.12$ ).
Bacteroidaceae spp. are primarily colonic bacteria; thus, the specificity of this relationship to fecal samples likely reflects growth in their preferred niche.

At the genus level, Bacteroides were strongly correlated with Escherichia-Shigella in feces in a nutritional status-dependent manner (Fig. 5c, d). Bacteroides were also strongly correlated with Salmonella in stunted children from the Afribiota dataset (Fig. 5e-h). Like E. coli, Salmonella has been previously shown to benefit from the presence of $B$. thetaiotaomicron. We confirmed that, like our E. coli strains, S. enterica Typhimurium benefited from the presence of the full Bacteroidales mix (Supplementary Fig. 7A) and provided a reciprocal advantage to Bacteroidales (Supplementary Fig. 7B) in MAL-M media. Salmonella was not detected in the other human studies that we analyzed, and other Enterobacteriaecae that were sporadically detected across studies (Klebsiella, Citrobacter, Enterobacter, and Providencia) were not significantly associated with Bacteroides in any of the datasets.

Linear growth stunting can be the result of many different dietary insufficiencies. To explicitly test the importance of iron insufficiency, we compared taxonomic correlations by stunting and anemia in children from Madagascar and CAR (the only populations for which anemia data was available). In this sub-population of two countries, the taxonomic correlation was again modulated by stunting status (stunted children: $n=152, P=0.0002$, rho $=0.29$; nonstunted children: $n=162, P=0.2175$, rho $=0.10$ ). Interestingly, however, Bacteroiaceae and Enterobacteriaceae were also significantly correlated in nonstunted anemic children $(n=44, P=0.03$, rho $=0.32)$. When malnourished children were defined as either stunted or anemic, the association between these taxa was highly significant ( $n=196, P=9.5 \mathrm{e}-06$, rho $=0.31$ ), while the slight trend seen in nonstunted children disappeared entirely in children who were neither stunted nor anemic $(n=112, P=0.9925$, rho $=-0.001)$. The modulation of this relationship by anemia is consistent with our in vitro observations that iron limitation contributes to codependencies between Bacteroidaceae and Enterobacteriaceae.

To further investigate the relationship between Bacteroidaceae and Enterobacteriaceae members at a species and functional level, we gathered publicly available fecal metagenomics data for stunted and nonstunted children 2-5 years old. We identified and processed datasets meeting these criteria from China ${ }^{29}$ and Zimbabwe ${ }^{30}$. Sample sizes after exclusion of younger children and of children without either Bacteroidaceae or Enterobacteriaceae present in the sample were modest $(N=7$ and 13 children, respectively, from China and Zimbabwe). Encouragingly, however, correlations between Bacteroidaceae and Enterobacteriaceae abundance in this small metagenomics dataset were consistent with those observed previously (Supplementary Table 3) and reached significance in stunted children from Zimbabwe and in the combined metagenomics abundance data (Supplementary Table 3; $N=20$, rho $=0.47, P=0.036$ ). We, therefore, proceeded with an exploratory analysis of species- and gene-level correlations in the pooled metagenomics dataset.

At the species level, the Bacteroides most strongly correlated with $E$. coli abundance were $B$. ovatus (rho $=0.48, P=0.031$ ) and $B$. dorei (rho $=0.44, P=0.049$ ). Indeed, as expected based on the family- and genus-level correlations, the majority of Bacteroides species were positively correlated with $E$. coli, and many of these species-level associations were consistent between datasets (Supplementary Table 4). These correlations should be interpreted with caution due to the small sample sizes; however, they suggest that in complex human microbiotas, $B$. ovatus and $B$. dorei cooperate particularly successfully with $E$. coli. This is consistent with our in vitro experiments in which both of these species showed cooperative individual growth with $E$. coli.

To explore gene-level associations, we binned all genes from the positively correlated Bacteroides species in Supplementary 

Table 1 Summary of two-sided Spearman's correlations between Bacteroidaceae and Enterobacteriaceae log-adjusted relative
abundance in 16S rRNA sequencing datasets, in samples where both taxa are present.

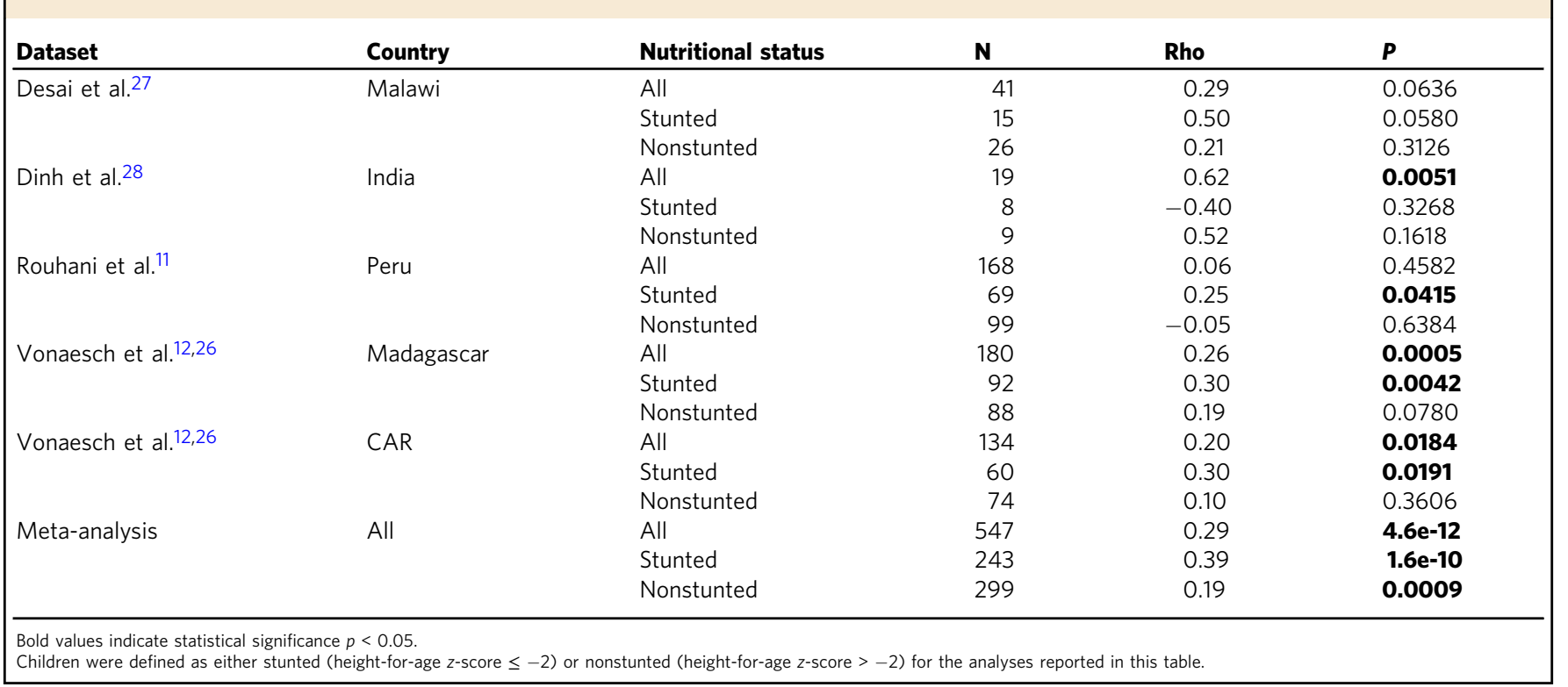

Table 4, reasoning there may be functional redundancy within this taxonomic group, and searched for any Bacteroides gene that correlated significantly with the abundance of $E$. coli or Enterobacteriaceae. Interestingly, an iron storage protein from the Bacteroides group was amongst the five genes correlated (raw $P$ value $<0.05$ ) with Enterobacteriaceae abundance (Supplementary Table 5). A larger number of genes were correlated with Escherichia abundance at the genus level, including multiple glycosidases for starch, cellulose, and host $\mathrm{N}$-glycan degradation, and several enzymes involved in B vitamin biosynthesis (Supplementary Table 6). Reciprocally, the only E. coli gene correlated with Bacteroides or Bacteroidaceae abundance was succinyl-diaminopimelate desuccinylase, a gene involved in both amino acid and cell wall biosynthesis (Supplementary Table 5). Although these correlations should all be interpreted with caution given the small sample size and a large number of genes (which prohibited correction for multiple testing), the functions identified are consistent with an exchange of nutrients between these taxa in malnourished environments.

Taken together, our data suggest that Bacteroidaceae and Enterobacteriaceae co-vary in the fecal microbiota of undernourished but not normally nourished children, consistent with the ability of these taxa to grow synergistically in carbohydraterich, protein-deficient environments (Fig. 6). Importantly, this relationship extends to pathogenic Enterobacteriaceae such as Salmonella. Moreover, it is highly consistent across independent human populations from seven different countries spanning three continents. This association may therefore be relevant to the growth of pathogens and pathobionts in the undernourished human intestine.

\section{Discussion}

Microbial communities are built from complex ecological interactions. Many of these interactions are negative: bacteria compete with one another and take advantage of their neighbors in the battle for scarce resources 31,32 . This high level of competition within the gastrointestinal microbiota is beneficial to the host, by maintaining relatively stable and diverse communities and by competitively excluding pathogens ${ }^{31-33}$. In contrast, bacterial cooperation is a relatively unusual ecological interaction with predicted destabilizing effects on microbial communities. By mutually enhancing one another's growth, cooperative bacteria are expected to 'boom and bust' together, leading to unstable and low-diversity communities ${ }^{31}$ which are susceptible to external species invasion ${ }^{34}$. Such low-diversity communities are generally considered to be the hallmark of dysbiosis in gut microbiota research, particularly when the high-abundance taxa are facultatively anaerobic Proteobacteria ${ }^{35}$. Here we find that a mixture of Bacteroidales and Enterobacteriaceae are capable of mutually cooperative, synergistic growth during undernutrition, leading to the outgrowth of a low-diversity community (Fig. 6).

The nutritional context was key to the emergence of synergistic growth in these bacteria: co-culture synergy required the presence of both host mucin and dietary carbohydrates, which Bacteroidales utilized to liberate a wide range of simple sugars. In contrast, synergistic growth was limited when protein or organic iron was abundant. Growth conditions favoring co-dependency are thus more likely to occur in the intestinal microbiota during protein undernutrition, where there is a paucity of dietary protein and iron compared to plant-based carbohydrates ${ }^{1,15}$. Indeed, we found that Bacteroidaceae and Enterobacteriaceae were strongly associated in the fecal microbiota of stunted or anemic children, but not in normally nourished children, indicating a nutrientdependent co-abundance pattern in the human intestine.

Increased abundance of Enterobacteriaceae members has been associated with child undernutrition across multiple studies ${ }^{10-13,36,37}$. Importantly, overgrowth of both Enterobacteriaceae and Bacteroidales has significant potential to contribute to intestinal damage and growth stunting. The murine experiment for this has already been done, showing that intestinal inflammation and exacerbated growth stunting occur in malnourished mice exposed to the same community of Bacteroidales and E. coli used in this study ${ }^{15}$. Healthy control mice exposed to these bacteria, or malnourished mice exposed only to Bacteroidales or to E. coli alone, did not develop intestinal damage. Members of the Bacteroidales have been repeatedly associated with a lean, non-obese phenotype in Western individuals ${ }^{38-40}$ and alleviate obesity in mice fed high-fat diets, by decreasing total energy harvest and by influencing host appetite and metabolism ${ }^{41}$. These same characteristics could be harmful to undernourished individuals struggling to gain weight. Moreover, in stunted children, 
(a) Severely Stunted

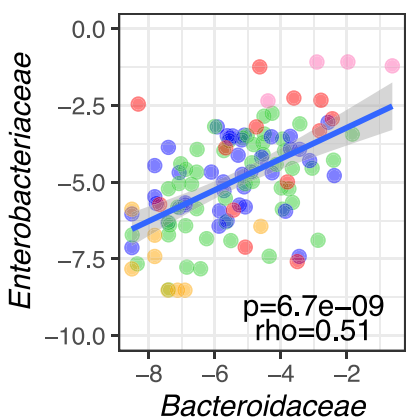

(c)

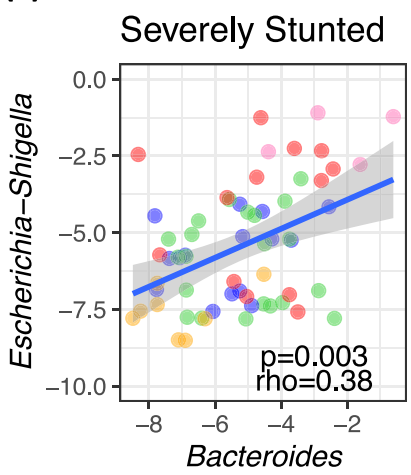

Healthy

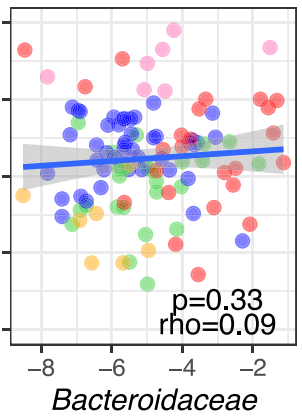

Bacteroidaceae

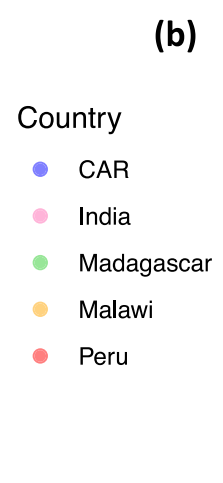

(d)

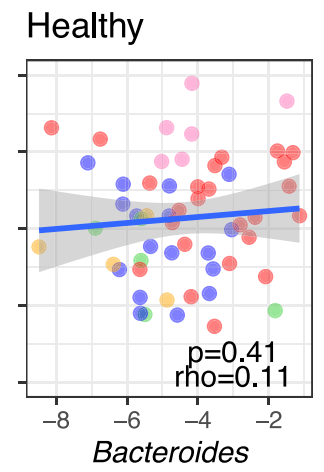

Country

- CAR

- India

- Madagascar

Malawi

Peru
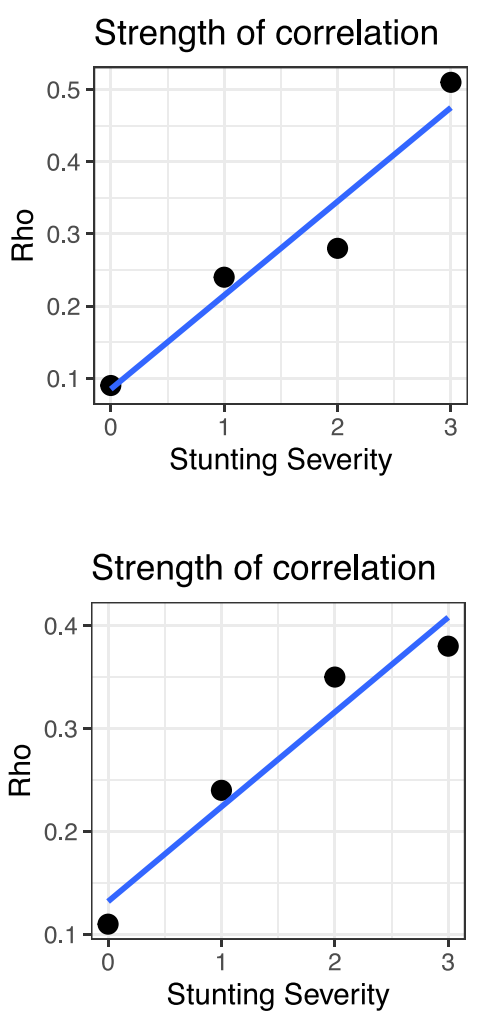

(f)

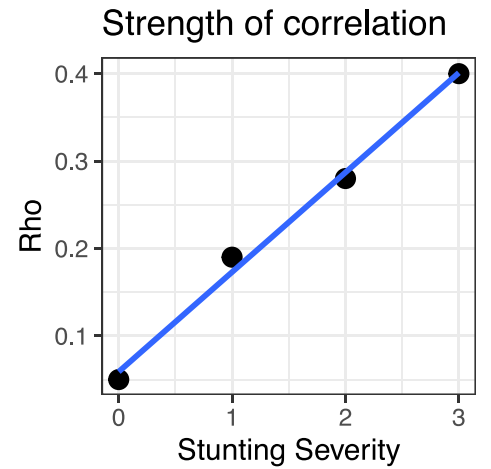

Fig. 5 Bacteroidaceae and Enterobacteriaceae are co-abundant in the fecal microbiota of undernourished children. a Correlation between the logtransformed relative abundance of Bacteroidaceae and Enterobacteriaceae in severely stunted $(n=115)$ and healthy children $(n=110)$. $\mathbf{b}$ The coefficient of correlation (Spearman's rho) as calculated between Bacteroidaceae and Enterobacteriaceae for each subset of stunting severity. c Correlation between the log-transformed relative abundance of Bacteroides and Escherichia-Shigella in severely stunted $(n=61)$ and healthy children $(n=56)$. d The coefficient of correlation (Spearman's rho) as calculated between Bacteroides and Escherichia-Shigella for each subset of stunting severity. e Correlation between the logadjusted relative abundance of Bacteroides and Salmonella in severely stunted $(n=67)$ and healthy children $(n=59)$. $\mathbf{f}$ The coefficient of correlation (Spearman's rho) as calculated between Bacteroides and Salmonella for each subset of stunting severity. All panels report two-sided Spearman's rho and $P$ value and exclude children for which one or both taxa were absent. The blue lines in (a-f) are a linear fit and the shaded areas in (a, $\mathbf{c}$, and $\mathbf{e})$ represent 95\% confidence interval. Stunting is defined as follows: 3 , "severely stunted" (HAZ $\leq-3) ; 2$, "moderately stunted" ( $-3>\mathrm{HAZ} \leq-2)$; 1 , "at risk of stunting" $(-2>\mathrm{HAZ} \leq-1)$, and 0 , "healthy" (HAZ $>-1)$.

B. fragilis, E. coli, Salmonella, and other members of the Enterobacteriaceae frequently carry virulence factors that contribute to intestinal inflammation and damage, thereby exacerbating growth stunting $10,16,36,42$.

It was recently shown that metabolic cross-feeding between intestinal commensals in Drosophila supported a mutual growth advantage for bacteria on malnourished diets, with the benefit for host reproduction ${ }^{43}$. The authors suggested a "buffering" effect whereby cross-feeding between commensals allows them to remain resilient to dietary insufficiencies ${ }^{43}$. As noted above, however, cooperative microbial communities also tend to be low-diversity and more susceptible to the invasion of novel species. Indeed, deliberate caloric restriction in humans was recently shown to reduce colonization resistance, leading to the expansion of $C$. difficile ${ }^{44}$. The fecal microbiota of malnourished children been found to include an overabundance of oral and upper airway bacteria, as well as a chronic overabundance of pathogens ${ }^{12}$; these patterns are consistent with reduced colonization resistance of the microbial ecosystem during child malnutrition, and we suggest this might be driven in part by destabilizing cooperative growth. Notably, an intervention trial that administered resistant-starch prebiotics to children in Malawi found that the intervention 


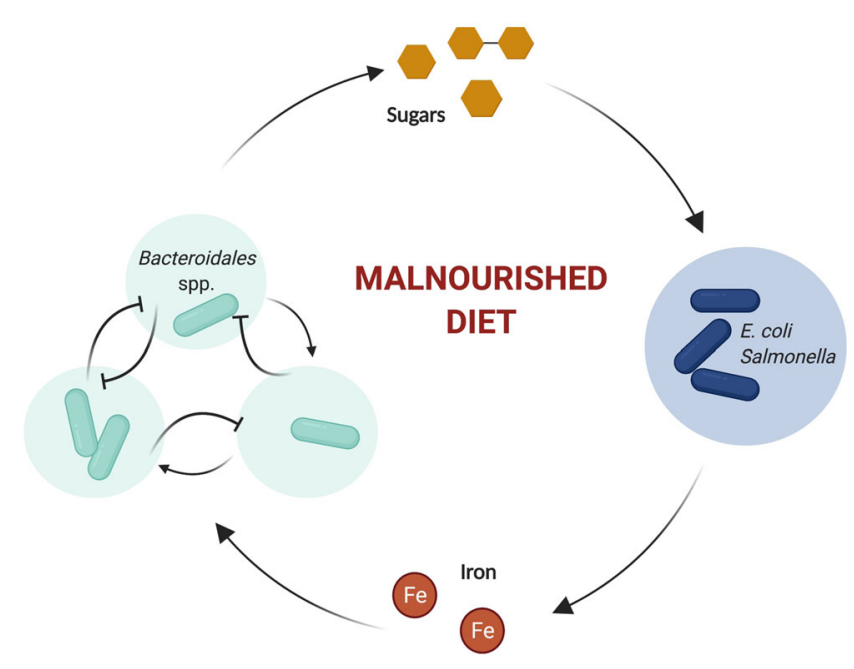

Fig. 6 Schematic of the metabolic network between Bacteroidales and

Enterobacteriaceae. In the context of a carbohydrate-rich, protein-deficient malnourished diet, Bacteroidales spp. cooperatively liberate sugars. Enterobacteriaceae members consume these sugars and provide iron to Bacteroidales. Together these interactions constitute a feedback loop leading to enhanced growth of both Enterobacteriaceae and Bacteroidales. These taxonomic groups contain notable pathogens, including $S$. enterica, enteroaggregative $E$. coli, and enterotoxin-producing B. fragilis, which may adversely affect intestinal health.

caused an increase in the fecal inflammatory marker calprotectin, as well as intestinal blooms in fecal Bacteroidetes, LPS biosynthesis, and specific E. coli-associated enzymes ${ }^{45}$. Although these results were unexpected and unfortunate, they are consistent with our prediction that an overabundance of fermentable fiber in malnourished children can exacerbate Bacteroides-E. coli cooperative growth and lead to intestinal inflammation.

It has been previously shown that certain Bacteroides spp. can support the growth of pathogenic E. coli and Salmonella ${ }^{17,18}$, and that certain E. coli strains can support Bacteroides growth ${ }^{21,22}$; however, the possibility for bidirectional feedback has never been explored. We find that many different Bacteroidales members are capable of growing synergistically with $E$. coli in low-nutrient conditions. Cooperative growth involved the exchange of soluble nutrients, including mucin- and carbohydrate-derived sugars and organic iron. Sialic acid was one such contributing sugar, consistent with previous reports ${ }^{17,18}$; however, a number of additional mono- and di-saccharides were produced by Bacteroidales in this nutritional context and supported E. coli growth. Human metagenomics analysis also supported the presence of carbohydrate cross-feeding, as multiple fiber- and sugar-degrading genes from Bacteroides spp. correlated with E. coli abundance in the microbiomes of stunted children.

Reciprocally, E. coli improved iron bioavailability for Bacteroidales in vitro, which might happen in a variety of ways: Enterobacteriaceae are known to secrete siderophores, degrade hemoglobin, and synthesize heme de novo ${ }^{22,24}$. Correlations between these taxa in the human microbiota was modulated by anemic status, suggesting that iron availability also plays an important role in cooperation in vivo. Furthermore, a Bacteroides spp. iron storage gene was one of the most strongly correlated with Enterobacteriaceae abundance in metagenomes from stunted children. Notably, iron usage by the microbiota has been shown to exacerbate anemia in mice, by directly competing with the host for iron uptake; the iron cross-feeding described here may therefore have additional consequences for host health ${ }^{46}$. It is also likely that other mechanisms not explored here, such as the exchange of amino acids and B vitamins, contribute to the synergistic growth advantage of these taxa in vitro and in vivo. Our gene-level analysis in humans was limited by the small number of available samples; further metagenomic sequencing efforts in populations of malnourished children would help to clarify some of these relationships.

Undernutrition is associated with significant microbial dysbiosis, but the factors driving this remain unclear; moreover, existing nutritional and sanitary interventions have failed to reverse child undernutrition. Both Bacteroidetes and Enterobacteriaceae have the potential to exacerbate EED and growth faltering in undernourished children, and have been shown to do so in mice. Here, we show that mutual growth synergy occurs between these taxa in protein- and iron-limited environments, underlying their co-occurrence in the gut microbiota of undernourished children. These data define a complex inter-species network with functional relevance to the undernourished gut and highlight a mechanism by which pathogen overgrowth might arise during undernutrition.

\section{Methods}

Bacterial strains and mutant construction. All bacterial strains, mutants, and plasmids used in this study are listed in Supplementary Table 2. B. fragilis wild-type strain $608 \mathrm{R}$ and $\triangle f e o A B$ were a generous gift from Dr. Edson Rocha, East Carolina University ${ }^{25}$. E. coli $\Delta$ nanA and $\Delta f u c I$ mutants were generated using homologous recombination to replace the target gene with a kanamycin resistance cassette $\left(\mathrm{Kan}^{\mathrm{R}}\right)$ following a protocol based on lambda red recombination ${ }^{47}$. Briefly, the $\mathrm{Kan}^{\mathrm{R}}$ locus on pKD13 (Supplementary Table 2) was PCR-amplified using primers with $50 \mathrm{bp} 5$ ' overhangs homologous to the target gene (Supplementary Table 7). Amplicons were purified by phenol chloroform extraction and electroporated into target E. coli carrying pkD46 expressing the lambda red recombinase. Transformants were selected on LB agar with $40 \mu \mathrm{g} / \mathrm{mL}$ kanamycin. Mutants were genotypically confirmed by PCR using primers flanking $\mathrm{Kan}^{\mathrm{R}}$. Mutant phenotypes were confirmed by aerobic growth in M9 minimal media with fucose or sialic acid as a sole carbon source. Growth was assessed by $\mathrm{OD}_{600}$ using a plate reader (Tecan).

Bacterial media and growth conditions. Bacteroidales and E. coli strains were routinely grown on Fastidious Anaerobic Agar (FAA) for $48 \mathrm{~h}$ at $37^{\circ} \mathrm{C}$ and in Fastidious Anaerobic Broth (FAB) for $24 \mathrm{~h}$ at $37^{\circ} \mathrm{C}$ with shaking at $250 \mathrm{rpm}$, under anaerobic conditions created by a sealed anaerobic jar and GasPak (BD). E. coli mutant strains were routinely grown with the addition of $40 \mu \mathrm{g} / \mathrm{mL}$ kanamycin and B. fragilis $\triangle f e o A B$ was routinely grown with the addition of $10 \mu \mathrm{g} / \mathrm{mL}$ erythromycin.

Complex media for growth experiments were prepared according to the macronutrient quantities shown in Supplementary Table S1. In addition, all complex media contained, per $1 \mathrm{~L}: 13.6 \mathrm{~g}$ of $\mathrm{KH}_{2} \mathrm{PO}_{4} ; 0.875 \mathrm{~g}$ of NaCl$; 1.125 \mathrm{~g}$ of $\left(\mathrm{NH}_{4}\right)_{2} \mathrm{SO}_{4} ; 1.0 \mathrm{~mL}$ of histidine-hematin solution $(1.9 \mathrm{mM}$ hematin in $0.2 \mathrm{M} \mathrm{L}$ histidine); $1.0 \mathrm{~mL} \mathrm{MgCl}_{2}$ solution ( $0.1 \mathrm{M}$ in water); $1.0 \mathrm{~mL}$ vitamin $\mathrm{K}_{3}$ solution $(1 \mathrm{mg} / \mathrm{mL}$ in ethanol); $1.0 \mathrm{~mL} \mathrm{CaCl}$ solution $(0.8 \% \mathrm{w} / \mathrm{v}$ in water); and $0.5 \mathrm{~mL}$ of vitamin $\mathrm{B}_{12}$ solution $(0.01 \mathrm{mg} / \mathrm{mL}$ in water). Where specified, additional histidine, hematin or vitamin B12 was added to MAL-M media during preparation, to achieve tenfold the normal concentration $(10 \mathrm{~mL}$ of histidine-hematin solution per $\mathrm{L}$, and $5 \mathrm{~mL}$ of vitamin B12 solution). Media $\mathrm{pH}$ was adjusted to 7.2 with concentrated $\mathrm{NaOH}$. Mucin was Type II Mucin from Porcine Stomach (PGM) (Sigma-Aldrich).

Bacteroidales-E. coli community growth assays. Overnight cultures of Bacteroidales and E. coli in FAB were resuspended and washed twice in a carbon-free buffer (either PBS-/- or minimal media containing no carbon source) and normalized to $\mathrm{OD}_{600}=0.35$ in PBS. These normalized strains were then combined in equal volumes to prepare inoculation mixtures, with any missing strains replaced by the equivalent volume in PBS. Within an anaerobic chamber, $70 \mu \mathrm{L}$ of bacterial inoculum was then added per $2 \mathrm{~mL}$ of degassed complex media, and co-cultures were grown anaerobically at $37^{\circ} \mathrm{C}$ for $24 \mathrm{~h}$ with shaking. To assess growth outcomes, samples were serially diluted in PBS, plated, and grown on LB agar aerobically at $37^{\circ} \mathrm{C}$ for $24 \mathrm{~h}$ to quantify E. coli growth, on FAA plates anaerobically to estimate total community growth, or on antibiotics-containing plates to enumerate mutant growth as appropriate. In the absence of a selective medium for all $\mathrm{Bac}$ teroidales species, Bacteroidales growth was quantified by qPCR. In all, 4-8 individual replicates are typically shown per growth assay, as stated in the figure legends for each panel. Each data point represents a single bacterial culture tube; further, each growth assay was repeated at least twice (i.e., from different seed cultures on a different day) with pooled results from all replicates shown. All media comparison experiments (Fig. 2 and Supplementary Fig. 2) were repeated at least three separate times with pooled results shown. The strain-wise replicates shown in 
Supplementary Fig. 3F-H (pooled in Supplementary Fig. 3D) represent a further level of replication, in that each "replicate" was performed by a different person using a different media batch; however each replicate still represents $\geq 8$ bacterial tubes over at least two separate growth curve days.

16S rRNA qPCR to assess bacterial growth. The total abundance of Bacteroidales and E. coli was assessed using taxa-specific primers (Supplementary Table 7 and the Quantitect SYBR-Green Mastermix (Qiagen) following manufacturer's protocols. Culture samples were heat-lysed at $100^{\circ} \mathrm{C}$ for $15 \mathrm{~min}$ to release DNA. qPCR was performed on cell lysates diluted 1:10, using an Applied Biosystems ${ }^{\mathrm{TM}} 7500$ Fast Real-Time PCR Machine. $\mathrm{C}_{\mathrm{T}}$ values were converted to $16 \mathrm{~S}$ copy numbers using a standard curve generated from $\mathrm{qPCR}$ amplification of purified $B$. thetaiotaomicron DNA or purified E. coli DNA. Results were expressed in $16 \mathrm{~S}$ copies $/ \mu \mathrm{L}$ of culture.

E. coli growth assessment in Bacteroidales supernatant. Endpoint cultures $(24 \mathrm{~h})$ of Bacteroidales or E. coli in MAL-M were centrifuged for $20 \mathrm{~min}$ at $16,000 \times g$, and the resultant supernatants were filter-sterilized through a $0.22-\mu \mathrm{m}$ filter. Fresh overnight cultures of $E$. coli were then normalized to $\mathrm{OD}_{600}=0.35$ as described above and were subcultured 1:200 into a final volume of $200 \mu \mathrm{L}$ of culture supernatant in a 96-well plate and grown anaerobically at $37^{\circ} \mathrm{C} . \mathrm{OD}_{600}$ was recorded every $15 \mathrm{~min}$ for $24 \mathrm{~h}$ using a plate reader (BioTek).

Siderophore assay. Siderophore production was assayed via Chrome Azurol S (CAS) Plate Assay as previously reported ${ }^{48}$. Briefly, CAS agar plates for siderophore detection were prepared using acid-washed glassware according to the following recipe: $50 \mathrm{~mL}$ of CAS $(30.25 \mathrm{mg} / \mathrm{mL})$ was mixed with $10 \mathrm{~mL}$ of iron(III) solution (containing $160 \mu \mathrm{l}$ of $6.25 \mathrm{M}$ hydrochloric acid and $27.03 \mathrm{mg}$ of $\mathrm{FECl}_{3} \cdot 6 \mathrm{H}_{2} \mathrm{O}$ per $100 \mathrm{~mL}$ ) and $40 \mathrm{~mL}$ of hexadecyltrimethyl ammonium bromide $(1.82 \mathrm{mg} / \mathrm{mL})$ and autoclaved. $100 \mathrm{~mL}$ of sterile CAS solution was then added to $900 \mathrm{~mL}$ freshly autoclaved minimal media (containing $1.0 \mathrm{~g}$ ammonium chloride, $1.704 \mathrm{~g} \mathrm{Na}_{2} \mathrm{HPO}_{4}, 1.089 \mathrm{~g} \mathrm{KH}_{2} \mathrm{PO}_{4}, 5.0 \mathrm{~g}$ sodium chloride, $30.24 \mathrm{~g}$ PIPES, $15 \mathrm{~g}$ agar, $1 \mathrm{~mL}$ of $1 \mathrm{M}$ magnesium sulfate and $10 \mathrm{~mL}$ of $50 \%$ glucose). Overnight cultures of E. coli were grown on a low-iron succinate minimal media ${ }^{49}$. These Cultures were normalized to an O.D.600 of 0.3 and $3 \mu \mathrm{L}$ of normalized culture were spotted onto a CAS indicator plate, for a total of nine spots per bacteria per plate. The negative control plate was $9 \times 3 \mu \mathrm{L}$ of the succinate minimal media. Plates were imaged after $24 \mathrm{~h}$ of incubation at $37^{\circ} \mathrm{C}$.

Metabolomics analysis. Bacterial culture supernatants were obtained at $16 \mathrm{~h}$ and $24 \mathrm{~h}$ from $2 \mathrm{~mL}$ bacterial cultures as described above and were frozen at $-70^{\circ} \mathrm{C}$ prior to analysis. Low molecular weight sugars and NAc-sugar amines were quantified by LC-MRM/MS at The Metabolomics Innovation Centre (TMIC) commercial service (University of Victoria, Genome BC Proteomics Centre), according to previously published UPLC-MRM/MS methods ${ }^{50}$, with necessary modifications. In brief, a mixed stock solution of 13 low-MW sugars and $4 \mathrm{~N}$-acetyl sugar amines was prepared with the use of their standard substances in $80 \%$ methanol at $500 \mu \mathrm{M}$ for each compound. This solution was then serially diluted with the same solvent to prepare calibration solutions in a concentration range of 0.002 to $125 \mu \mathrm{M}$. For chemical derivatization, $20 \mu \mathrm{L}$ of each medium sample or each calibration solution was mixed in turn with $20 \mu \mathrm{L}$ of an internal standard solution containing ${ }^{13} \mathrm{C}_{6}$-glucose, ${ }^{13} \mathrm{C}_{6}$-mannose, ${ }^{13} \mathrm{C}_{6}$-fructose, and ${ }^{13} \mathrm{C}_{5}$-ribose in water, $40 \mu \mathrm{L}$ of $200 \mathrm{mM} 3$-nitrophenylhydrazine hydrochloride solution in $60 \%$ methanol and $40 \mu \mathrm{L}$ of $200 \mathrm{mM}$ EDC. $\mathrm{HCl}$ solution prepared in a mixed solvent of methanol/water/pyridine $(60: 40: 5, \mathrm{v} / \mathrm{v} / \mathrm{v})$. The mixture was allowed to react at $50{ }^{\circ} \mathrm{C}$ for $90 \mathrm{~min}$. After the reaction, $20-\mu \mathrm{L}$ aliquots were injected onto a Phenomenx PFP UPLC column $(2.1 \times 150 \mathrm{~mm}, 1.7 \mu \mathrm{m})$ to run LC-MS/MS on an Agilent 1290 UHPLC system coupled to an Agilent 6495B QQQ mass spectrometer, which was operated in the negative-ion multiple-reaction monitoring (MRM) mode. For LC separation, $0.1 \%$ formic acid in water/methanol $(76 / 24, \mathrm{v} / \mathrm{v})$ was used as the mobile phase at $0.25 \mathrm{~mL} / \mathrm{min}$ and $40^{\circ} \mathrm{C}$ for isocratic elution over a period of $15 \mathrm{~min}$. The raw data were acquired using the Agilent MassHunter ${ }^{\oplus} 7.0$ software. After data acquisitions, linearly regressed calibration curves of individual compounds were constructed with the analyte-to-internal standard peak area ratios measured from the injection of the calibration curves. For those compounds without their isotopelabeling analogs as the internal standards, ${ }^{13} \mathrm{C}_{6}$-fructose was used a common internal standard. Concentrations of the analytes were calculated by interpolating the calibration curves of individual compounds with their analyte-to-internal standard peak area ratios measured from the injection of the sample solutions

Quantification of short-chain fatty acids was performed in-house according to a method developed by Han et al., with minor modification ${ }^{51}$. Briefly, $500 \mu \mathrm{L}$ of supernatant were mixed with $500 \mu \mathrm{L}$ of $50 \%$ acetonitrile, then the mixture was vortexed for $5 \mathrm{~min}$ and centrifuged at $7000 \times g$ for $5 \mathrm{~min}$. In total, $50 \mu \mathrm{L}$ of the organic phase were derivatized adding $20 \mu \mathrm{L}$ of $200 \mathrm{mM} 3 \mathrm{NPH}$ in $50 \%$ acetonitrile and $20 \mu \mathrm{L}$ $120 \mathrm{mM}$ EDC solution of $6 \%$ pyridine in $50 \%$ acetonitrile. The mixture was left under agitation at $40^{\circ} \mathrm{C}$ for $30 \mathrm{~min}$. After this time reaction was stopped adding $100 \mu \mathrm{L}$ of $0.1 \%$ formic acid in $90 \%$ acetonitrile. All samples were injected into the mass spectrometer right after being processed. An LC-MS/MS system that was equipped with an Agilent Technologies 1200 high-performance liquid chromatography (HPLC) instrument, an Agilent 6460 triple quadrupole (QQQ) mass spectrometer, and a reversed-phase column Zorbax $300 \mathrm{C} 18250 \times 4.6 \mathrm{~mm}$ (Agilent Technologies) was employed. MS was conducted in positive ion mode with an electrospray ionization voltage of $3500 \mathrm{~V}$ while using $50 \mathrm{psi}$ nebulizer gas at a temperature of $350^{\circ} \mathrm{C}$. The sample injection volume was $5 \mu \mathrm{L}$. LC separation was performed while using mobile phase A $(0.1 \%$ formic acid in $3 \%$ acetonitrile $97 \%$ water $)$ and mobile phase B $(0.1 \%$ formic acid in $90 \%$ acetonitrile), at a flow rate of $650 \mu \mathrm{L} / \mathrm{min}$. The separation gradient was as follows: $20 \%$ B for 3 min., $20-30 \%$ B in $1 \mathrm{~min}$. In all, $30-40 \%$ B in 7 min., $40-50 \%$ in $2 \mathrm{~min}$., $50-100 \%$ in $2 \mathrm{~min}$., $100 \%$ for $1 \mathrm{~min}$., $100-20$ in $1 \mathrm{~min}$., and $20 \%$ for $2 \mathrm{~min}$. Collision energy of $10 \mathrm{~V}$ was used for multiple-reaction monitoring (MRM), and LC-MS/MS data were analyzed by Mass Hunter Qualitative Analysis B.06.00 software (Agilent Technologies). The identification and quantification of the SCFAs were carried out based on the retention time and mass fragmentation pattern compared with standards. Six-point calibration curves made by peak area vs concentration of the pure standards were used to quantify the different SCFA. The linearity of the curves was determined by the coefficient of determination $\left(R^{2}\right)$, being higher than 0.99 for all standards. Concentrations of the SCFAs were calculated by interpolating the calibration curves of individual compounds.

High-throughput $16 \mathrm{~S}$ sequencing for analysis of community growth kinetics Culture samples were collected at 0,16 , and $24 \mathrm{~h}$ from bacteria grown in MAL-M as described above, and samples were heat-lysed at $100^{\circ} \mathrm{C}$ for $15 \mathrm{~min}$. The $16 \mathrm{~S}$ rRNA gene (V4 region) was amplified directly from lysates using Illumina-tagged, barcoded primers $515 \mathrm{~F}-806 \mathrm{R}^{49}$. Reactions were purified and normalized using the 96-well Sequal-Prep kit. Amplicons were pooled and gel-purified using a GeneJet Gel Extraction Kit (ThermoFisher) to remove primer dimers, and DNA yield was quantified by PicoGreen and qPCR. The final library was pooled and sequenced on an Illumina MiSeq platform with $30 \%$ PhiX, using the v2 kit for paired-end 250 bp reads.

Bioinformatic analysis of in vitro bacterial community. Demultiplexed forward reads were analyzed in QIIME2 ${ }^{52}$, using the DADA2 option for sequence quality control $^{53}$ and trimming to $250 \mathrm{bp}$. Taxonomic assignment was performed using a Naive Bayes classifier trained on the SILVA database (v132, 99\% OTU sequences, region 515F/806R) ${ }^{54}$. For Bacteroides which were not identified to the species level by the SILVA database, the $16 \mathrm{~S}$ sequence was further queried using BLAST to rationally assign all five BG community members. Filtering at the amplicon sequence variant (ASV) level was performed in R using the phyloseq package ${ }^{55}$, to remove any ASVs that were not assigned to the initial community members $(4.8 \%$ of all reads). Taxonomic barplots were then created using ggplot $2^{56}$. To calculate the total abundance of each individual Bacteroidales species over time, relative abundances were multiplied by the total Bacteroidales abundance, as determined by $16 \mathrm{~S}$ Bacteroidetes-specific qPCR, at each time point.

Bioinformatic analysis of human fecal microbiota: $16 \mathrm{~S}$ amplicon sequencing $16 \mathrm{~S}$ rRNA gene sequencing of the V4 region were retrieved from four studies ${ }^{11,12,28,57}$ spanning five countries (Madagascar, Central African Republic, India, Malawi, and Peru) and included both publicly available data as well as new sequences available through the Afribiota study.

Children were included for analysis if they were 2-5 years of age and had linear growth measurements (height- or length-for-age- $z$-score) available as an outcome. Where longitudinal samples were available, a single sample at 2 years of age was included per child. For Dihn et al. 2016, height-for-age $z$-score was calculated from available height, age, and gender data using the public World Health Organization tool $^{58}$. In all datasets, stunting was defined as HAZ $\leq-2$ and nonstunted as $H A Z>-2$. To further bin samples by stunting, we defined stunting severity as follows: 3 , "severely stunted" (HAZ $\leq-3) ; 2$, "moderately stunted" $(-3>\mathrm{HAZ} \leq-2)$; 1 , "at risk of stunting" $(-2>\mathrm{HAZ} \leq-1)$, and 0 , "healthy" (HAZ $>-1)$. Anemia was defined by a blood hemoglobin level of less than $11 \mathrm{~g} / 100 \mathrm{~mL}$ after adjustment for altitude (hemoglobin levels in Madagascar were adjusted by $-0.2 \mathrm{~g} / 100 \mathrm{~mL}$ to account for the height above sea level, while hemoglobin levels in Central African Republic were approximately at sea level and were unadjusted $)^{59}$.

Data from the Afribiota study were generated by co-authors Vonaesch PV and Sansonetti $\mathrm{PJ}^{12}$. The study protocol for Afribiota was approved by the Institutional Review Board of the Institut Pasteur (2016-06/IRB), the National Ethical Review Boards of Madagascar (55/MSANP/CE) and the Central African Republic (173/ UB/FACSS/CSCVPER/16), and the Human Ethics Board of the University of British Columbia (H18-01108), in accordance with the principles of the Declaration of Helsinki. All participants received oral and written information about the study and the legal representatives of the children provided written consent to participate in the study ${ }^{26}$. The detailed inclusion and exclusion criteria and recruitment procedures of the Afribiota study are described in the published protocol ${ }^{26}$. Part of the dataset was previously published and is available at the European Nucleotide Archive (ENA) accession PRJEB27868. New sequencing data analyzed here are deposited at the ENA accession PRJEB48119.

To obtain new sequences through the Afribiota study, DNA was extracted with a QiaAmp cador ${ }^{\circledR}$ Pathogen Mini kit using an additional bead-beating step and library preparation was performed as recommended by Kozich et al. ${ }^{41}$ using standard barcoded primers targeting the 16S V4 region (v4.SA501- v4.SA508 and v4.SA701- v4.SA712). The amplicon library was sequenced on a MiSeq using the 
MiSeq 500 Cycle V2 Reagent Kit $(250 \times 2)$. OTU table was generated using the Dada2 pipelines developed by the Holmes lab with slight modifications (https:// benjjneb.github.io/dada2 $/{ }^{62}$ ) and taxonomy was assigned using the Silva Database (version 132). The OTU table was transferred to Huus KE for further analysis.

Data from Desai et al. were obtained directly as an OTU table from their publicly available GitHub page (https://github.com/chandnil77/

BacterialViralMicrobiome_of_GrowthVelocity_in_EED) ${ }^{27}$. Raw sequencing data from Dinh et al. ${ }^{28}$ and Rouhani et al. ${ }^{11}$ were downloaded from the NCBI Sequence Read Archive (accession codes PRJNA279828 and PRJEB28159 respectively) using SRAtoolkit (v 2.10.8) and analyzed in qiime2 using the Deblur error correction option and SILVA taxonomic assignment (99\% OTU sequences, region 515F/806R) ${ }^{52,60}$. Further processing of all studies was performed in $\mathrm{R}$, including exclusion of taxa with low fractional abundance $(<0.001 \%)$ and rarefaction to 5000 reads. Samples that were missing either taxon (i.e., zero values) were excluded for correlation analysis, and the relative abundance of nonzero values was log-transformed.

Bioinformatic analysis of human fecal microbiota: shotgun sequencing. Shotgun metagenomics sequencing data of the human fecal microbiome were retrieved from two published studies in Zimbabwe and China ${ }^{29,30}$ that reported linear growth data in children. Children were included for analysis if they were 2-5 years of age and had linear growth measurements (HAZ) available as an outcome.

Raw sequencing data were retrieved from the NCBI Nucleotide Sequence Archive (accession codes PRJNA521455 and PRJNA543967) using SRAtoolkit (v 2.10.8) and processed according to the published Biobakery workflow (https:// huttenhower.sph.harvard.edu/biobakery_workflows/ $)^{61}$. Sequence quality control and removal of human reads was performed using KneadData 0.7.4; bacterial abundances were calculated using MetaPhlan3.0; and bacterial gene abundances were calculated with Humann3 using nucleotide-based alignment against the Chocophlan database ${ }^{62}$. Gene abundance tables were grouped by uniref90_level4ec and normalized to copies per million (cpm) according to the standard pipeline.

Normalized taxa and gene abundance tables were imported into R. Taxonomic data were processed analogously to the $16 \mathrm{~S}$ data using Phyloseq ${ }^{55}$, as described above and as detailed in the attached $\mathrm{R}$ markdown file. Also as described above, stunting was defined by HAZ, and samples that were missing either taxon (i.e., zero values) were excluded for correlation analysis. Species-level analysis was performed by pairwise Spearman's correlation of all detected Bacteroides species against the abundance of $E$. coli, and associations were reported by raw $P$ value for taxa that were consistent (positively or negatively associated) in both datasets individually. For subsequent gene-level analysis, genes from all Bacteroides species that were consistently positively associated with $E$. coli were binned together (i.e., summed per gene) and the gene table was further filtered for genes present in at least $60 \%$ (12 out of 20) samples, to try and reduce sparseness of the dataset while maintaining sample size. Within this subset of genes, pairwise Spearman's correlations against the abundance of Enterobacteriaceae and Escherichia were performed, and both raw $P$ value and FDR-corrected $P$ value are reported. In the reciprocal analysis, annotated $E$. coli genes were filtered for genes present in at least $60 \%$ of samples and pairwise Spearman's correlations were again performed against the abundance of Bacteroidaceae and Bacteroides.

Summary figures. Summary figures (Figs. 1a and 6) were created with BioRender.com.

Statistical analysis. Statistical analysis was performed in GraphPad Prism (www.graphpad.com) (v 9.1.1) or in R Studio (v 1.2.1335) using the packages phyloseq (v 1.32.0), ggplot2 (v 2.3.3.2), dplyr (v 1.0.2), tidyr (v 1.1.2), vegan (v 2.5.6), RColorBrewer ( $\mathrm{v} 1.1 .2$ ), biomformat ( $\mathrm{v}$ 1.16.0), reshape ( $\mathrm{v}$ 2.1.4.4), and psych ( $\mathrm{v}$ 2.0.8). Unless otherwise indicated, aggregate results represent the mean $+I-$ SEM, and statistical significance is represented by $* P$ value $<0.05$, ${ }^{*} P$ value $<0.01,{ }^{* * *} P$ value $<0.001$ and ${ }^{* * * *} P$ value $<0.0001$. For in vitro experimental work, parametric tests were chosen based on the normal distribution of bacterial growth outcomes. Student's two-sided $t$ test was applied for comparisons of two groups; ANOVA for comparisons involving three or more groups; and twoway ANOVA for grouped analyses, for example, all analyses comparing single and co-culture across different media conditions. The post hoc test for ANOVA (Sidak, Tukey, or Dunnett) was chosen based on Prism's default statistical recommendation, which takes into account the number of comparisons being tested. For human microbiota data analysis, nonparametric Spearman's correlations were chosen based on the non-normal distribution of relative abundance data. Sample size, center and dispersion metrics, and statistical tests are reported in the figure legends. Exact $P$ values and test statistics for all statistical results presented in the main and supplemental figures are listed in Supplementary Table 8.

Reporting summary. Further information on research design is available in the Nature Research Reporting Summary linked to this article.

\section{Data availability}

New 16S rRNA sequencing data generated through the Afribiota project has been deposited to the ENA under accession PRJEB48119. Part of the Afribiota dataset was previously published and is available at the European Nucleotide Archive (ENA) accession PRJEB27868. All other sequencing data used in the study were previously published. Data from Desai et al. ${ }^{27}$ were obtained directly as an OTU table from their publicly available GitHub page (https://github.com/chandni177/

BacterialViralMicrobiome of GrowthVelocity in EED). 16S rRNA sequencing data from Dinh et al. ${ }^{28}$ were retrieved from the NCBI Sequence Read Archive (SRA) under accession code PRJNA279828. 16S rRNA sequencing data from Rouhani et al. ${ }^{11}$ were retrieved from the NCBI SRA under accession code PRJEB28159. Shotgun metagenomics sequencing data from Osakunor et al. ${ }^{30}$ were retrieved from the NCBI SRA under accession code PRJNA521455. Shotgun metagenomics sequencing data from Li et al. ${ }^{29}$ were retrieved from the NCBI SRA under accession code PRJNA543967. The SILVA database v132 for $16 \mathrm{~S}$ taxonomic assignment can be accessed from https://www.arbsilva.de/download/archive/qiime/. The Chocophlan database for metagenomics assignment was accessed from https://github.com/biobakery/humann\#download-thechocophlan-database. Raw metabolomics data have been deposited at Metabolomics Workbench accession number PR001175. Reagents and bacterial strains are available upon request. Source data are provided with this paper.

\section{Code availability}

Code is available at https://github.com/khuus/Finlay_Public.

Received: 3 December 2020; Accepted: 8 November 2021; Published online: 25 November 2021

\section{References}

1. Black, R. E. et al. Maternal and child undernutrition and overweight in lowincome and middle-income countries. Lancet 382, 427-451 (2013).

2. Popkin, B. M., Corvalan, C. \& Grummer-Strawn, L. M. Dynamics of the double burden of malnutrition and the changing nutrition reality. Lancet 395, 65-74 (2020).

3. Lindenbaum, J. et al. Subclinical small-intestinal disease in East Pakistan. BMJ 2, 1616-1619 (1966)

4. Lindenbaum, J. Recovery of small-intestinal structure and function after residence in the tropics: I. studies in peace corps volunteers. Ann. Intern. Med. 74, 218 (1971).

5. Kelly, P. et al. Responses of small intestinal architecture and function over time to environmental factors in a tropical population. Am. J. tropical Med. Hyg. 70, 412-419 (2004).

6. Kelly, P. et al. Endomicroscopic and transcriptomic analysis of impaired barrier function and malabsorption in environmental enteropathy. PLOS Neglected Tropical Dis. 10, e0004600 (2016).

7. Denno, D. M. et al. Use of the lactulose to mannitol ratio to evaluate childhood environmental enteric dysfunction: a systematic review. Clin. Infect. Dis. 59, S213-S219 (2014).

8. Campbell, D. I., Elia, M. \& Lunn, P. G. Growth faltering in rural Gambian infants is associated with impaired small intestinal barrier function, leading to endotoxemia and systemic inflammation. J. Nutr. 133, 1332-1338 (2003).

9. Campbell, D. I., McPhail, G., Lunn, P. G., Elia, M. \& Jeffries, D. J. Intestinal inflammation measured by fecal neopterin in Gambian children with enteropathy: association with growth failure, Giardia lamblia, and intestinal permeability. J. Pediatr. Gastroenterol. Nutr. 39, 153-157 (2004).

10. Kosek, M. N. et al. Causal pathways from enteropathogens to environmental enteropathy: findings from the MAL-ED birth cohort study. eBioMedicine https://doi.org/10.1016/j.ebiom.2017.02.024 (2017).

11. Rouhani, S. et al. Gut microbiota features associated with campylobacter burden and postnatal linear growth deficits in a Peruvian birth cohort. Clin. Infect. Dis. https://doi.org/10.1093/cid/ciz906 (2019).

12. Vonaesch, P. et al. Stunted childhood growth is associated with decompartmentalization of the gastrointestinal tract and overgrowth of oropharyngeal taxa. Proc. Natl Acad. Sci. USA 115, E8489-E8498 (2018).

13. Subramanian, S. et al. Persistent gut microbiota immaturity in malnourished Bangladeshi children. Nature https://doi.org/10.1038/nature13421 (2014).

14. Cumming, O. et al. The implications of three major new trials for the effect of water, sanitation and hygiene on childhood diarrhea and stunting: a consensus statement. BMC Med. 17, 173 (2019).

15. Brown, E. M. et al. Diet and specific microbial exposure trigger features of environmental enteropathy in a novel murine model. Nat. Commun. 6, 7806 (2015).

16. Kau, A. L. et al. Functional characterization of IgA-targeted bacterial taxa from undernourished Malawian children that produce diet-dependent enteropathy. Sci. Transl. Med. 7, 276ra24 (2015).

17. Ng, K. M. et al. Microbiota-liberated host sugars facilitate post-antibiotic expansion of enteric pathogens. Nature 502, 96-99 (2013) 
18. Huang, Y.-L., Chassard, C., Hausmann, M., von Itzstein, M. \& Hennet, T. Sialic acid catabolism drives intestinal inflammation and microbial dysbiosis in mice. Nat. Commun. 6, 8141 (2015).

19. Charbonneau, M. R. et al. Sialylated milk oligosaccharides promote microbiota-dependent growth in models of infant undernutrition. Cell https:// doi.org/10.1016/j.cell.2016.01.024 (2016).

20. Grondin, J. M., Tamura, K., Déjean, G., Abbott, D. W. \& Brumer, H. Polysaccharide utilization loci: fueling microbial communities. J. Bacteriol. 199, e00860-16 (2017).

21. Otto, B. R., van Dooren, S. J. M., Dozois, C. M., Luirink, J. \& Oudega, B. Escherichia coli hemoglobin protease autotransporter contributes to synergistic abscess formation and heme-dependent growth of Bacteroides fragilis. Infect. Immun. 70, 5-10 (2002).

22. Zhu, W. et al. Xenosiderophore utilization promotes Bacteroides thetaiotaomicron resilience during colitis. Cell Host Microbe 27, 376-388.e8 (2020).

23. Sperry, J. F., Appleman, M. D. \& Wilkins, T. D. Requirement of heme for growth of Bacteroides fragilis. Appl. Environ. Microbiol. 34, 386-390 (1977)

24. Halpern, D. \& Gruss, A. A sensitive bacterial-growth-based test reveals how intestinal Bacteroides meet their porphyrin requirement. BMC Microbiol. 15, 1-9 (2015).

25. Rocha, E. R., Bergonia, H. A., Gerdes, S. \& Jeffrey Smith, C. Bacteroides fragilis requires the ferrous-iron transporter FeoAB and the CobN-like proteins BtuS1 and BtuS2 for assimilation of iron released from heme. Microbiologyopen 8, e00669 (2019).

26. Vonaesch, P. et al. Identifying the etiology and pathophysiology underlying stunting and environmental enteropathy: study protocol of the AFRIBIOTA project. BMC Pediatr. 18, 236 (2018)

27. Desai, C. et al. Growth velocity in children with environmental enteric dysfunction is associated with specific bacterial and viral taxa of the gastrointestinal tract in Malawian children. PLoS Negl. Trop. Dis. 14, e0008387 (2020).

28. Dinh, D. M. et al. Longitudinal analysis of the intestinal microbiota in persistently stunted young children in South India. PLOS ONE 11, e0155405 (2016).

29. Li, D. et al. Intestinal Bacteroides sp. imbalance associated with the occurrence of childhood undernutrition in China. Front. Microbiol. 10, 2635 (2019).

30. Osakunor, D. N. M. et al. The gut microbiome but not the resistome is associated with urogenital schistosomiasis in preschool-aged children. 1. Commun. Biol. 3, 1-11. (2020).

31. Coyte, K. Z., Schluter, J. \& Foster, K. R. The ecology of the microbiome: networks, competition, and stability. Science 350, 663-666 (2015).

32. Coyte, K. Z. \& Rakoff-Nahoum, S. Understanding competition and cooperation within the mammalian gut microbiome. Curr. Biol. 29 R538-R544 (2019).

33. Foster, K. R., Schluter, J., Coyte, K. Z. \& Rakoff-Nahoum, S. The evolution of the host microbiome as an ecosystem on a leash. 7665. Nature 548, 43-51 (2017).

34. Machado, D. et al. Polarization of microbial communities between competitive and cooperative metabolism. Nat. Ecol. Evol. 5, 195-203 (2021).

35. Tiffany, C. R. \& Bäumler, A. J. Dysbiosis: from fiction to function. Am. J. Physiol. Gastrointest. Liver Physiol. 317, G602-G608 (2019).

36. Rogawski, E. T. et al. Epidemiology of enteroaggregative Escherichia coli infections and associated outcomes in the MAL-ED birth cohort. PLoS Neglected Tropical Dis. 11, e0005798 (2017).

37. Khan Mirzaei, M. et al. Bacteriophages isolated from stunted children can regulate gut bacterial communities in an age-specific manner. Cell Host Microbe 27, 199-212.e5 (2020).

38. Wang, K. et al. Parabacteroides distasonis alleviates obesity and metabolic dysfunctions via production of succinate and secondary bile acids. Cell Rep. 26, 222-235.e5 (2019).

39. Yang, J.-Y. et al. Gut commensal Bacteroides acidifaciens prevents obesity and improves insulin sensitivity in mice. Mucosal Immunol. 10, 104-116 (2017).

40. Ridaura, V. K. et al. Gut microbiota from twins discordant for obesity modulate metabolism in mice. Science 341, 1241214 (2013).

41. Johnson, E. L., Heaver, S. L., Walters, W. A. \& Ley, R. E. Microbiome and metabolic disease: revisiting the bacterial phylum Bacteroidetes. J. Mol. Med 95, 1-8 (2017).

42. Wagner, V. E. et al. Effects of a Gut Pathobiont in a Gnotobiotic Mouse Model of Childhood Undernutrition (Washington University in St. Louis, 2017).

43. Henriques, S. F. et al. Metabolic cross-feeding in imbalanced diets allows gut microbes to improve reproduction and alter host behaviour. 1. Nat. Commun. 11, 4236 (2020).

44. von Schwartzenberg, R. J. et al. Caloric restriction disrupts the microbiota and colonization resistance. Nature https://doi.org/10.1038/s41586-021-03663-4 (2021).
45. Ordiz, M. I. et al. The effect of dietary resistant starch type 2 on the microbiota and markers of gut inflammation in rural Malawi children. Microbiome 3, 1-9 (2015).

46. Das, N. K. et al. Microbial metabolite signaling is required for systemic iron homeostasis. Cell Metab. 31, 115-130.e6 (2020).

47. Datsenko, K. A. \& Wanner, B. L. One-step inactivation of chromosomal genes in Escherichia coli K-12 using PCR products. Proc. Natl Acad. Sci. USA 97, 6640-6645 (2000).

48. Schwyn, B. \& Neilands, J. B. Universal chemical assay for the detection and determination of siderophores. Anal. Biochem. 160, 47-56 (1987).

49. Tostado-Islas, O. et al. Iron limitation by transferrin promotes simultaneous cheating of pyoverdine and exoprotease in Pseudomonas aeruginosa. ISMEJ. 15, 2379-2389 (2021).

50. Han, J., Lin, K., Sequria, C., Yang, J. \& Borchers, C. H. Quantitation of low molecular weight sugars by chemical derivatization-liquid chromatography/ multiple reaction monitoring/mass spectrometry. Electrophoresis 37, 1851-1860 (2016).

51. Han, J., Lin, K., Sequeira, C. \& Borchers, C. H. An isotope-labeled chemical derivatization method for the quantitation of short-chain fatty acids in human feces by liquid chromatography-tandem mass spectrometry. Analytica Chim. Acta 854, 86-94 (2015).

52. Bolyen, E. et al. Reproducible, interactive, scalable and extensible microbiome data science using QIIME 2. Nat. Biotechnol. 37, 852-857 (2019).

53. Callahan, B. J. et al. DADA2: high resolution sample inference from Illumina amplicon data. Nat. Methods 13, 581 (2016).

54. Quast, C. et al. The SILVA ribosomal RNA gene database project: improved data processing and web-based tools. Nucleic Acids Res. 41, D590-D596. (2012).

55. McMurdie, P. J. \& Holmes, S. phyloseq: an R package for reproducible interactive analysis and graphics of microbiome census data. PLOS ONE 8, e61217 (2013)

56. Wickham, H. ggplot2: Elegant Graphics for Data Analysis (Springer-Verlag, 2016).

57. Desai, M. S. et al. A dietary fiber-deprived gut microbiota degrades the colonic mucus barrier and enhances pathogen susceptibility. Cell 167, 1339-1353.e21 (2016).

58. Bloem, M. The 2006 WHO child growth standards. BMJ 334, 705-706 (2007).

59. World Health Organization. Haemoglobin Concentrations for the Diagnosis of Anaemia and Assessment of Severity (WHO, 2011).

60. Amir, A. et al. Deblur rapidly resolves single-nucleotide community sequence patterns. mSystems 2, e00191-16 (2017).

61. Beghini, F. et al. Integrating taxonomic, functional, and strain-level profiling of diverse microbial communities with bioBakery 3. eLife 10, e65088 (2021).

62. Franzosa, E. A. et al. Species-level functional profiling of metagenomes and metatranscriptomes. Nat. Methods 15, 962-968 (2018)

\section{Acknowledgements}

Many thanks to Dr. Cara Haney for her feedback on early versions of this story and to Dr. Jon Briggs for his helpful input on Bacteroides carbohydrate metabolism. We are also grateful to the authors of Dinh et al. 2016, Desai et al. 2020, Vonaesch et al. 2018, Rouhani et al. 2019, Li et al. 2019, and Osakunor et al. 2020 for making their data and metadata freely available, and to Dr. Edson Rocha for generously sending us the $B$. fragilis $\triangle f e o A B$ mutant strain. We would also like to thank our anonymous reviewers for their valuable feedback and suggestions. Work in B.B.F.'s lab was funded by grants from Canadian Institutes for Health Research. B.B.F. is also a Senior Fellow at the Canadian Institute for Advanced Research. K.E.H. and M.C. were supported by a CIHR Vanier Scholarship and a UBC Four-Year Fellowship. T.T.H. was supported by a Faculty of Medicine Summer Student Research Scholarship and by a Canadian Association of Gastroenterology Undergraduate Research Scholarship. S.L.V. was supported by a Killam Postdoctoral Research Fellowship, a Michael Smith Foundation for Health Research Postdoctoral Fellowship, and a CIHR Postdoctoral Fellowship. P.V. was supported by a Swiss National Science Foundation Early and Advanced Postdoctoral Fellowship, a RouxCantarini Postdoctoral Fellowship, and a Swiss National Science Foundation Return Grant

\section{Author contributions}

K.E.H. designed the study, conducted the experiments, analyzed the data, and wrote the paper. T.T.H. conducted the experiments, analyzed the data, and wrote the paper. A.C.C., M.C., S.L.V., and K.K.J. conducted experiments, assisted with the methodology, and edited the paper. P.V. and P.J.S. generated the data from the Afribiota study and edited the paper. B.B.F. supervised the work and edited the paper.

\section{Competing interests}

The authors declare no competing interests. 


\section{Additional information}

Supplementary information The online version contains supplementary material available at https://doi.org/10.1038/s41467-021-27191-x.

Correspondence and requests for materials should be addressed to B. B. Finlay.

Peer review information Nature Communications thanks the anonymous reviewer(s) for their contribution to the peer review of this work.

Reprints and permission information is available at http://www.nature.com/reprints

Publisher's note Springer Nature remains neutral with regard to jurisdictional claims in published maps and institutional affiliations. (c) (1) Open Access This article is licensed under a Creative Commons Attribution 4.0 International License, which permits use, sharing, adaptation, distribution and reproduction in any medium or format, as long as you give appropriate credit to the original author(s) and the source, provide a link to the Creative Commons license, and indicate if changes were made. The images or other third party material in this article are included in the article's Creative Commons license, unless indicated otherwise in a credit line to the material. If material is not included in the article's Creative Commons license and your intended use is not permitted by statutory regulation or exceeds the permitted use, you will need to obtain permission directly from the copyright holder. To view a copy of this license, visit http://creativecommons.org/ licenses/by/4.0/.

(C) The Author(s) 2021 